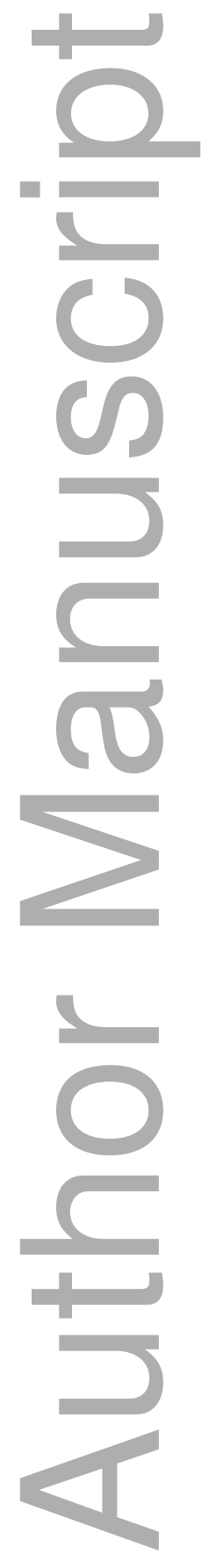

This is the author manuscript accepted for publication and has undergone full peer review but has not been through the copyediting, typesetting, pagination and proofreading process, which may lead to differences between this version and the Version of Record. Please cite this article as doi: $\underline{10.1111 / P 0 M S .13060}$

This article is protected by copyright. All rights reserved 


\title{
Service Level Constrained Inventory Systems
}

\author{
Yuchen Jiang \\ Department of Industrial \& Operations Engineering, University of Michigan, Ann Arbor, MI, ycjiang@umich.edu \\ Cong Shi* (Corresponding Author) \\ Department of Industrial \& Operations Engineering, University of Michigan, Ann Arbor, MI, shicong@umich.edu \\ Siqian Shen \\ Department of Industrial \& Operations Engineering, University of Michigan, Ann Arbor, MI, siqian@umich.edu
}

Motivated by the importance of service quality in nowadays customer business environment, we focus on inventory optimization under probabilistic service level constraints, namely, the $\alpha$ service level (also known as the ready rate) or the $\beta$ service level (also known as the fill rate). Under service level constraints, we consider two canonical stochastic inventory models: (i) the classical inventory control model with backlogging and (ii) the remanufacturing inventory control model with random product returns. The random demands could be nonstationary, evolving and correlated over time. For each model, we first establish the optimality of generalized base-stock policies, and then propose a new approximation algorithm that admits a worst-case performance guarantee of 2 . The core concept developed in this paper is called the delayed forced holding and production cost, which is proven effective in dealing with service level constrained inventory systems. We also provide an efficient heuristic algorithm for the multi-item inventory system. Our extensive computational experiments show that the proposed algorithms perform within $2 \%$ of optimality.

Key words: inventory; service level; remanufacturing; optimal policies; approximation algorithms

Received: March 2018; Accepted: April 2019 by Qi Annabelle Feng after one revision.

\section{Introduction}

In nowadays customer-driven business environment, it is vital for companies to focus on the quality of service (QoS) when managing operations and businesses. Since the early 2000s, firms started to put tremendous effort and resource into understanding the customers and markets. Those who could consistently provide superior service to their customers would remain an excellent reputation and keep most of their loyal buyers. Customers facing stockouts have been observed abandoning their purchases, switching retailers, substituting similar items and have seldom gone back (see, e.g., Fitzsimons (2000)). One of the most common challenges in making supply chain decisions, at its most fundamental, boils down to minimizing inventory control cost while still delivering high-quality customer service, which we are interested in modeling and solving in this paper.

On one hand, the notion of service level requirement has been widely used both in theory and in practice to measure the performance of inventory replenishment policies (cf. Ghiani et al. 2005).

This article is protected by copyright. All rights reserved 
It is typically defined as a probabilistic constraint so that the demand is satisfied with a high probability. By enforcing a service level requirement, companies are able to improve the QoS by guaranteeing a small stockout rates. There are several empirical studies of the sensitivity of inventory service levels on demand in business-to-consumer settings (cf. Fitzsimons 2000, Anderson et al. 2006, Jing and Lewis 2011). In particular, according to Jing and Lewis (2011), stockout rates have a significant impact on the firm's profitability and the firm can achieve many of the benefits through small decreases in stockout rates.

There are abundant examples in practice where the service level plays an important role in firms' supply chain management. For example, the grocery store industry has generally a very high service level expectation, especially for its dairy product section. A customer who wants to buy $2 \%$ reduced-fat milk should find it with a very high probability. If not, the store runs the risk of losing the sale as well as the customer. Clearly, customers are more willing to buy from those grocery stores who always have enough stock. This directly explains why the Kroger Co., one of the country's largest supermarket chains, generally enforces a high service level (from $85 \%$ to $98 \%$ ) on dairy products. Their optimal inventory replenishment policy must meet the service level requirement while minimizing the total inventory cost over the planning horizon. Likewise, many other industries, such as food and fashion, also set a high service level that helps satisfy customers' demands and avoid stockouts. In addition, service level agreements (SLA) are usually enforeed in some industries such as the semi-conductor industry to guarantee the delivery of manufactured products. As pointed out by Katok et al. (2008), SLAs are used to improve supply chain coordination and there are contractual financial penalties and rewards associated with failing or achieving a target service level. In general, having a service level requirement helps firms maintain their reputation and increase their revenue in the long run (see Chen and Krass (2001) for more examples and discussions).

On the other hand, after-sales services could also be crucial in delivering great customer services (cf. Cohen et al. 2006). Companies have to handle the return, repair, and disposal of failed components. The returned products, though some parts may be damaged, can be remanufactured and resold. The remanufacturing process includes repair or replacement of worn-out or obsolete components and modules, which has a lower production cost than the manufacturing process. Examples of remanufacturing occur in many industries, such as personal computers, cell phones, automotive parts, etc. For example, Cummins Inc., an American corporation that manufactures and distributes engines, filtration, and power generation products, has more than 45 years of remanufacturing experience. The firm remanufactures engines and other automobile parts by replacing all wear items with new parts. In addition, Cummins also imposes a high service level requirement on their

\section{This article is protected by copyright. All rights reserved}


long-term supply, as it cannot afford to lose customers. The company is much more willing to make extra productions and hold them as inventory than having a lost-sales which may potentially jeopardize their reputation. Both the service level requirement and its remanufacturing process help Cummins Inc. building up a great reputation of customer service, which benefits the company in the long run.

To address all the aforementioned issues in inventory management, we study periodic-review service level constrained stochastic inventory systems where the stockout probability is lower bounded by a threshold value in each period. This type of service level constraint is commonly known as the $\alpha$ service level in the literature (see, e.g., Simchi-Levi et al. (2014), Snyder and Shen (2011), Chen and Krass (2001)). We consider two fundamental stochastic inventory models with $\alpha$ service level constraints: the multi-period backlogging model and the multi-period backlogging model with remanufacturing, with a general stochastic demand process (i.e., correlated, nonstationary and evolving demand). In the service level constrained backlogging model, the firm makes a production decision in each period to minimize the total expected production, holding and backlogging costs over a finite planning horizon, subject to a given service level requirement. In the counterpart model with remanufacturing, in addition to the regular production, there are some products being returned at the beginning of each period (commonly referred to as cores, see, e.g., Tao and Zhou (2014)), which can be remanufactured into regular products at a lower cost. The objective is to decide the manufacturing and remanufacturing quantities in each period so as to minimize the total expected costs, subject to a given service level requirement.

As seen from our literature review below, there has been growing research on both the theoretical and computational aspects of service level constrained inventory systems. There are mainly two sources of motivation. First, traditional inventory models usually assume linear cost functions to penalize inventory, backorders, or lost sales. However, the assumption of linear backlogging or lost-sale penalty is primary for analytical tractability rather than an accurate representation of reality (see Bertsimas and Paschalidis (2001) for a detailed discussion). The mechanism of varying unit penalty costs can hardly take effect on the QoS performance of a system, mainly due to the difficulty of quantifying customer satisfaction. In this regard, imposing a target service level is a much more direct way to quantify and improve the QoS performance of an inventory system. Second, as extensively discussed in Chen and Krass (2001), the backlogging cost is often very difficult to quantify in practice. Hence, a target service level constraint is thus considered as an effective (if not more so) alternative performance measure.

In this paper, we consider a generalized model that incorporates both the service level constraints and the penalty cost for each unsatisfied demand, which has several advantages. First, when the 
firm does not have a good estimate of the backlogging cost, the firm can simply set the per-unit penalty cost to be zero in our model, which then reduces to the conventional model with service level constraints only. Our algorithms, analysis, and results hold for this special case. Second, when the firm does have a good estimate of the backlogging cost (e.g., from historical data), considering backlogging cost together can significantly alleviate the problem of suffering from severe backlogs in the worst-case scenarios (since the service level constraints only guarantee that the demand will be met with a certain positive probability in each period).

\subsection{Main Results and Contributions}

We consider two canonical inventory systems under probabilistic level constraints: (i) the classical inventory control model with backlogging and (ii) the remanufacturing inventory control model with random product returns. The service level constraint can either be $\alpha$ type (ready rate) or $\beta$ type (fill rate). The random demand and return processes could be non-stationary, evolving and generally correlated. We summarize the key results and our contributions as follows.

(a) For the classical backlogging model, we establish the optimality of a base-stock policy (Proposition 1). For the remanufacturing model, we establish the optimality of a total base-stock policy (Proposition 2), i.e., in each period, we bring the total inventory position (after production) to an optimal threshold level by remanufacturing as many returned products as possible.

(b) Finding the exact optimal policy via a brute-force dynamic programming (DP) approach is computationally intractable, despite its simple form. We propose two efficient approximation algorithms, termed the Split-Merge-Balance (SMB) algorithm and the Modified Split-Merge-Balance $(M S M B)$ algorithm, for the backlogging model and the remanufacturing model, respectively. Our theoretical performance analysis shows that both algorithms admit a worst-case performance of two, i.e., the expected cost of our algorithm is at most twice the expected cost of an optimal solution (Theorems 1 and 2).

(c) Leveraging the ideas from the SMB algorithms, we also propose a heuristic algorithm for the multi-item inventory system under an aggregate service level. Through an extensive numerical study, we demonstrate that the proposed policies (SMB, MSMB, and the heuristic algorithm) perform within $2 \%$ of optimality and also yield a significant reduction of solution time.

The core new concept developed in this paper is the notion of delayed forced costs in designing provably-good policies for service level constrained inventory systems. The major difficulty of designing approximation algorithms for service level constrained models is the impossibility of balancing the expected marginal holding costs against the expected marginal backlogging costs,

\section{This article is protected by copyright. All rights reserved}


which is the dominated technique (i.e., cost-balancing technique) in some related literature (see, e.g., Levi et al. 2007, 2008a,b). Our algorithms first split the marginal costs into two parts (i.e., forced costs and future costs) and shift all the forced costs to one period later (called delayed forced costs); after regrouping the future costs and delayed costs according to their monotonicity, our algorithms balance the expected overage cost against the expected underage cost associated with each period. The introduction of the delayed forced costs makes the worst-case analysis invariably harder $\neg$ one needs to group consecutive intervals together to amortize the sum of future cost and delayed forced cost against the optimal policy (Lemma 3). This is in sharp contrast to the aforementioned studies where period-by-period amortization is sufficient for the classical backlogging model.

For the remanufacturing model, the amortization of production costs becomes non-trivial and our worst-case analysis builds upon on the elegant partitioning technique introduced in Tao and Zhou (2014). There is a challenge we need to overcome: due to the service level constraints in our model, we split each holding cost and production cost into two parts and use the delayed holding cost and delayed production cost when designing a modified SMB algorithm. Consequently, our worst-case analysis needs to bound both parts of production costs in different sets of periods, which cannot be readily adapted from their results (see the detailed discussions before Lemma 6).

In general, we believe that the concept of delayed forced costs could be widely applied in designing algorithms for any general service level constrained inventory systems.

\subsection{Literature Review}

This paper is closely related to the following research domains and related literature.

Stochastic inventory system with service level constraints. Bookbinder and Tan (1988) studied a probabilistic lot-sizing problem using a "static-dynamic uncertainty" strategy. In their two-stage model, a retailer first decided a schedule (or replenishment periods) to place orders. Then, the retailer made adjustments to the planned orders when demand was released. Chen and Krass (2001) showed that the $(s, S)$ policy is optimal under independently and identically distributed (i.i.d.) demands for an infinite-horizon stationary setting. Boyaci and Gallego (2001) proposed effective heuristic procedures to serial inventory systems with service level constraints on stockout probabilities. Shang and Song (2006) also studied a serial base-stock inventory model under simple Poisson demands and the same type of service level constraints. They developed a closedform approximation for the optimal base-stock levels. Bertsimas and Paschalidis (2001) considered a multiclass make-to-stock manufacturing system with probabilistic service level guarantees, and 
devised a production policy that minimized inventory costs under a stockout probability guarantee using queueing methods. Goh and Sim (2011) carried out a computational study (using a software called ROME) of a distributionally robust periodic-review inventory problem with fill rate constraints. More recently, Wei et al. (2018) studied a periodic-review service level constrained inventory system with lost-sales and lead times. They proposed a simple heuristic by solving a linear programming (LP) problem derived from a deterministic inventory model with backlogging, and showed that the proposed heuristic is asymptotically optimal as the service level grows to $100 \%$, and derived a simple and explicit bound on the optimality gap. The probabilistic constraints that impose service level guarantees in each period (i.e., $\alpha$ service levels) are used in the majority of the literature, which is also the primary focus of this paper.

Stochastic inventory system with remanufacturing. Zhou et al. (2011) studied the structure of optimal policies for the remanufacturing inventory system with multiple types of returned products. They showed that the optimal policy is a modified base-stock policy, which can be completely characterized by a sequence of control parameters. Tao and Zhou (2014) proposed an approximation algorithm for the stochastic inventory system with remanufacturing. They also proved that the cost of their proposed algorithm is at most twice of the optimal cost. Gong and Chao (2013) focused on the capacitated inventory systems with remanufacturing. Building upon the preservation result by Chen et al. (2013), they showed that the optimal remanufacturing policy is a modified remanufacture-down-to policy and the optimal manufacturing policy is a modified total-up-to policy. Our remanufacturing model differs from all of the aforementioned models by incorporating service level constraints in each period.

Approximation algorithms on inventory systems. The DP approach is effective in characterizing the structural properties of optimal policies. However, the computational complexity is very sensitive to the dimension of the state space. In fact, it has been shown in Halman et al. (2009) that the stochastic lot-sizing model (without service level constraints) is NP-hard. Our work is closely related to recent studies of approximation algorithms for stochastic periodic-review inventory systems pioneered by Levi et al. (2007). They first introduced the marginal cost accounting scheme, which associated a cost with each decision made by a particular policy. They proposed a dual-balancing policy which admitted a worst-case performance guarantee of 2 for the backlogging model. Subsequently, Levi et al. (2008a,b) and Levi and Shi (2013) proposed approximation algorithms for the lost-sales, capacitated, and lot-sizing models, respectively. More recently, Truong (2014) re-derived the 2-approximation ratio for the backlogging model via a look-ahead optimization approach. Tao and Zhou (2014) proposed an approximation algorithm for a remanufacturing system with a worst-case performance guarantee of two. Cheung et al. (2016), Nagarajan and Shi 
(2016) proposed approximation algorithms for submodular joint replenishment problems. There has also been a series of studies on perishable/fresh inventory systems (see, e.g., Chao et al. (2015, 2017), Zhang et al. (2016)). However, their models did not consider service level constraints while our paper focuses on designing approximation algorithms for models with service level guarantees.

\subsection{Structure of the Paper and General Notation}

The remainder of the paper is organized as follows. We formulate the service level constrained backlogging model as a dynamic program and present the structural properties of optimal policies in $\S 2$. We derive a 2-approximation algorithm for the classical backlogging model in $\S 3$. We extend our structural result to the remanufacturing system and also derive a 2-approximation algorithm in $\S 4$. We also give a heuristic algorithm to the multi-item setting in $\S 5$. We carry out numerical experiments and demonstrate the effectiveness of our proposed policy in $\S 6$. Finally, we conclude the paper and point out some future research avenues in $\S 7$.

Throughout the paper, we use increasing and decreasing in a non-strict sense. For notational convenience, we often use a capital letter and its lower-case form to distinguish between a random variable and its realization. We use LHS and RHS as abbreviations for the "left-hand side" and the "right-hand side" of an equation, respectively. We use $\triangleq$ to mean "is defined as", and $\mathbb{1}(A)$ is the indicator function taking value 1 if statement " $\mathrm{A}$ " is true and 0 otherwise. For any $x \in \mathbb{R}$, we denote $x^{+}=\max \{x, 0\}$. For any sequence $x_{i}, i=1,2, \ldots$, we let $x_{[i, j]}=\sum_{k=i}^{j} x_{k}$ and $x_{[i, j)}=\sum_{k=i}^{j-1} x_{k}$, where the summation over an empty set is defined as 0 . For any $a, b \in \mathbb{R}$, we denote $a \wedge b=\min \{a, b\}$.

\section{Periodic-Review Inventory Systems with Service Level Constraints}

Consider a finite planning horizon of $T$ periods indexed by $t=1, \ldots, T$. The production lead time is assumed to be $L \geq 0$.

Demand structure. We adopt the same demand structure as in Levi et al. (2007) and Tao and Zhou (2014). An information set $f_{t}$ is observed at the beginning of each period $t$. It contains all the available information that can be used to predict future demands, such as the realized demands $\left(d_{1}, \ldots, d_{t-1}\right)$ and possibly some other exogenous information (denoted by $\rho_{t}$ at period $t$ ). For example, when the state of economy is observed at each period, $\rho_{t}$ corresponds to the state of economy at period $t$. The conditional joint distribution of the future demands $\left(D_{t}, \ldots, D_{T}\right)$ is determined by $f_{t}=\left(d_{1}, \ldots, d_{t-1}, \rho_{1}, \ldots, \rho_{t}\right)$. We denote $\mathcal{F}_{t}$ as the set of all the possible realizations of the information set $f_{t}$. Our demand model generalizes the existing correlated demand models in the literature, such as Markov-modulated demand process (MMDP) (Sethi and Cheng 1997), 
autoregressive demand (Mills 1991), which will be described in $\S 6$. Our demand model is also useful in practice, in which martingale model of forecast evolution (MMFE for short, see, e.g., Graves et al. 1986, Heath and Jackson 1994) and advance demand information (ADI) (see, e.g., Gallego and Özer 2001) are used to forecast the future demand.

Service level requirements. Our model incorporates the service level requirement. There are mainly two types of service level constraints used in practice. The first one is the $\alpha$ type, also known as the ready rate, defined as a stockout probability for each period $t$. Following Chen and Krass (2001), the service level constraint enforces that the demand in each period $t+L$ is satisfied by a certain probability $\theta_{t}$ (which is in fact associated with period $t+L$ ), i.e.,

$$
\mathbb{P}\left(N I_{t+L} \geq 0 \mid f_{t}\right) \geq \theta_{t}, \quad \forall t=1, \cdots, T
$$

where $N I_{t}$ denotes the net inventory at the end of period $t$, which can be either positive (in the presence of on-hand inventory) or negative (in the presence of backorders). The ready rate has been considered by, e.g., Bookbinder and Tan (1988), Nahmias (1993), Sethi and Cheng (1997) and Chen and Krass (2001).

The second one is the $\beta$ type, also known as the fill rate, defined as the percentage of demand that is met from stock. More formally, given $\tau_{t} \in(0,1)$ as the target fill rate in period $t+L$, the fill rate constraint is given by

$$
\min \left\{1, \mathbb{E}\left[\frac{\left(N I_{t+L-1}+q_{t}\right)^{+}}{D_{t+L}} \mid f_{t}\right]\right\} \geq \tau_{t}, \quad \forall t=1, \ldots, T
$$

where $q_{t}$ is the total production quantity at period $t$. Note that practitioners often prefer using the fill rate over the ready rate since the fill rate is arguably much easier to evaluate.

Our service level constrained model is able to incorporate either constraint (1) or (2). Note that our production decisions will not affect net inventories for the first $L$ periods, and hence we enforce the service level requirements from period $L+1$ to $L+T$.

System dynamics. In each period $t$, events occur in the following sequence: First, the manufacturer determines the production quantity (denoted by $q_{t}$ ) in period $t$. The planned production quantity should satisfy the service level constraint (1) or (2). Then $d_{t}$ units of demands are received. As a consequence, unused products are stored as inventory, which incurs the holding cost; on the other hand, unsatisfied demands incur the backlogging cost and are carried to the next period. The production, holding and backlogging cost functions are assumed to be linear, with per-unit costs $c_{t}, h_{t}$ and $b_{t}$, respectively. The goal is to decide production quantities that achieve the required service level in each period and to minimize total expected cost at the same time. 
A dynamic programming formulation. We formulate the problem using dynamic programming (DP) approach. Since no products will arrive in the first $L$ periods, it suffices to consider the total cost from period $L+1$ to period $L+T$. We first calculate the immediate cost associated with decisions in period $t$. Define $x_{t}$ as the inventory position at the beginning of period $t$, which equals to the current on-hand inventory plus the pipeline inventory minus the backorders, i.e., $x_{t}=N I_{t-1}+q_{[t-L, t)}$. Then the inventory position in the next period equals to the current inventory position plus production quantity minus the demand in the current period, i.e., $x_{t+1}=x_{t}+q_{t}-d_{t}$. Let $y_{t}=x_{t}+q_{t} \geq x_{t}$ be the inventory position in period $t$ after production. Then the net inventory at the end of period $t+L$ can be written as $N I_{t+L}=y_{t}-D_{[t, t+L]}$. Therefore, the total holding and backlogging cost is given by

$$
G_{t}\left(y_{t}, f_{t}\right) \triangleq h_{t+L} \mathbb{E}\left[\left(y_{t}-D_{[t, t+L]}\right)^{+} \mid f_{t}\right]+b_{t+L} \mathbb{E}\left[\left(D_{[t, t+L]}-y_{t}\right)^{+} \mid f_{t}\right]
$$

It is clear that $G_{t}$ is continuous and convex in its first component. Adding the production cost, the total cost in period $t$ is $G_{t}\left(y_{t}, f_{t}\right)+c_{t} q_{t}$.

For the $\alpha$ service level constraints, we can rewrite (1) as

$$
\mathbb{P}\left(y_{t}-D_{[t, t+L]} \geq 0 \mid f_{t}\right) \geq \theta_{t}, \quad \forall t=1, \ldots, T
$$

To simplify the above constraint, we define a threshold value

$$
r_{t}^{1}=r_{t}^{1}\left(f_{t}\right) \triangleq \inf \left\{r \in \mathbb{R}^{+}: \mathbb{P}\left(D_{[t, t+L]} \leq r \mid f_{t}\right) \geq \theta_{t}\right\}
$$

i.e., $r_{t}^{1}\left(f_{t}\right)$ is the $\theta_{t}$-quantile of the random variable $D_{[t, t+L]}$ given information $f_{t}$. Then the service level constraint (3) is equivalent to $y_{t} \geq r_{t}^{1}$ for all $t=1, \ldots, T$. In some special cases, $r_{t}^{1}$ is very easy to compute. For example, when demands are independent Poisson or Normal random variables, the aggregate demand $D_{[t, t+L]}$ follows the same distribution type. For general demand distributions, we can use the Monte Carlo sampling method of Glasserman (2004) to empirically obtain $r_{t}^{1}$.

For the $\beta$ service level constraints, we can rewrite (2) as

$$
\mathbb{E}\left[\frac{\left(y_{t}-D_{[t, t+L)}\right)^{+}}{D_{t+L}} \mid f_{t}\right] \geq \tau_{t}, \quad \forall t=1, \cdots, T .
$$

Note that the LHS of (22) is non-decreasing in $y_{t}$, the above constraint can also be simplified to $y_{t} \geq r_{t}^{2}$ for all $t=1, \ldots, T$, where $r_{t}^{2}$ is a function in $f_{t}$ satisfying

$$
\mathbb{E}\left[\frac{\left(r_{t}^{2}-D_{[t, t+L)}\right)^{+}}{D_{t+L}} \mid f_{t}\right]=\tau_{t}
$$

In practice, the value of $r_{t}^{2}$ can be obtained via bisection search and Monte Carlo sampling of future demands $D_{t}, D_{t+1}, \ldots, D_{t+L}$. 
To summarize, both service level constaints can be equivalently simplified to a lower bound on the inventory position $y_{t}$, i.e., $y_{t} \geq r_{t}\left(f_{t}\right)$, for all $t=1, \ldots, T$. In each period $t=1, \ldots, T$, we can recompute $r_{t}, \ldots, r_{T}$ based on $f_{t}$, using simulation. Therefore, it is equivalent to imposing the lower bound constraints in our model, regardless of the type of service level constaints.

Let $v_{t}\left(x_{t}, f_{t}\right)$ be the minimal expected cost from period $t+L$ to period $T+L$ given the inventory position $x_{t}$ and the information set $f_{t} \in \mathcal{F}_{t}$ at the beginning of period $t$. The Bellman's equations are

$$
\begin{aligned}
& v_{T+1}\left(x_{T+1}, f_{T+1}\right)=0, \quad \forall x_{T+1} \in \mathbb{R}, f_{T+1} \in \mathcal{F}_{T+1}, \\
& v_{t}\left(x_{t}, f_{t}\right)=\min _{y_{t} \geq \max \left\{r_{t}, x_{t}\right\}}\left\{G_{t}\left(y_{t}, f_{t}\right)+c_{t} q_{t}+\mathbb{E}\left[v_{t+1}\left(y_{t}-D_{t}, \mathcal{F}_{t+1}\right) \mid f_{t}\right]\right\}, \quad t=1, \ldots, T .
\end{aligned}
$$

Structure of optimal policies. Using (5), the structure of optimal policies is characterized in Proposition 1. It is a special case of Proposition 2 and its proof can be found in Appendix A.

Proposition 1. For the inventory control problem defined in (5), an optimal policy is a modified base-stock policy. More specifically, there exists $\left\{s\left(f_{t}\right)\right\}_{t=1}^{T}$ such that

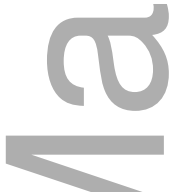

$$
y_{t}^{*}\left(x_{t}\right)= \begin{cases}\max \left\{r_{t}, s\left(f_{t}\right)\right\}, & \text { if } x_{t}<s\left(f_{t}\right) \\ \max \left\{r_{t}, x_{t}\right\}, & \text { if } x_{t} \geq s\left(f_{t}\right) .\end{cases}
$$

Proposition 1 asserts that any optimal policy has the following structure: if the inventory position in period $t$ is no less than the threshold $s\left(f_{t}\right)$, an optimal policy produces up to the service level $r_{t}$; otherwise, it brings the inventory position to $\max \left\{r_{t}, s\left(f_{t}\right)\right\}$. Therefore, the higher the service level, the more orders are placed by the optimal policy. In particular, when there is no service level requirement presented in the model (i.e., $r_{t}=0$ ), the structure of optimal policy reduces to the well-known base-stock policy (see, e.g., Zipkin (2000)).

\section{Provably-Good Policies for Service Level Constrained Inventory Systems}

Computing an exact optimal policy through a brute-force DP model is generally intractable under correlated demand structures, despite the simple structure of optimal policies. To this end, we provide an approximation algorithm, called Split-Merge-Balance policy (denoted by the SMB policy), for practically solving the service level constrained inventory problem. We show that the SMB policy admits a worst-case performance guarantee of 2, i.e., the expected cost of the policy is at most twice the expected cost of an optimal policy, regardless of any distributions of the random demands and choices of the cost parameters. Moreover, the SMB policy performs empirically near-optimal, demonstrated by extensive numerical tests in $\S 6$. 
Figure 1 Dual-balancing policy given by Levi et al. (2007) (no balancing point due to the service level constraint)

The traditional inventory cost accounting scheme (in DP) decomposes the total costs by periods. In the following, we present a new marginal cost accounting scheme for our model under service level constraints: it decomposes the total cost in terms of the marginal costs of individual decisions and these marginal costs may include costs in both the current and subsequent periods. This extends and generalizes the marginal cost accounting discussed by Levi et al. (2007).

\subsection{Review of the Dual-Balancing Policy}

The underlying idea of the SMB policy is based on the dual-balancing policy proposed by Levi et al. (2007). The traditional inventory cost accounting scheme (in dynamic programming) decomposes the total costs by periods; Levi et al. (2007) proposes a marginal cost accounting scheme and computes the marginal holding cost by

$$
H_{t}\left(q_{t}\right)=\sum_{j=t+L}^{T+L} h_{j}\left(\left(X_{t}+q_{t}-D_{[t, j]}\right)^{+}-\left(X_{t}-D_{[t, j]}\right)^{+}\right),
$$

where $X_{t}$ denotes a random inventory position which realizes at the beginning of period $t$.

The marginal backlogging cost is the same as the classical per-period backlogging cost, i.e.,

$$
\Pi_{t}\left(q_{t}\right)=b_{t+L}\left(D_{[t, t+L]}-\left(q_{t}+X_{t}\right)\right)^{+}
$$

The dual balancing policy balances the marginal holding cost in (6) against the marginal backlogging cost (7) and it admits a worst-case performance guarantee of two.

However, the dual balancing policy cannot be directly applied to our model because the balancing quantity for the marginal holding cost and the marginal backlogging cost may not exist due to the service level constraints. In periods where the constrained service level is much higher than the current inventory position, the expected marginal holding cost is always greater than the expected marginal backlogging cost. In such a case, one cannot find a feasible production quantity which balances the expected marginal holding cost against the expected marginal backlogging cost.

\subsection{Split-Merge-Balance Policy}

Without loss of generality, we assume that the unit production cost in each period is zero following a standard cost transformation in the literature (see, e.g., Zipkin (2000)). That is, for any system with positive unit production $\operatorname{cost} c_{t}$, there is an equivalent system with revised $\operatorname{costs} c_{t}^{\prime}=0$, 
$h_{t+L}^{\prime}=h_{t+L}+c_{t}-c_{t+1}$ and $b_{t+L}^{\prime}=b_{t+L}-c_{t}+c_{t+1}$. This allows us to only consider holding costs and backlogging costs.

Marginal cost accounting scheme (Split). We first present a new marginal cost accounting scheme for our inventory model under service level requirements, which generalizes the marginal cost accounting scheme discussed by Levi et al. (2007). In the presence of service level constraints, we split the marginal holding cost into two parts. The first part is called forced holding cost (denoted by $\tilde{H}_{t}$ ), which accounts for the holding cost from producing up to the service level $\bar{X}_{t}=\max \left\{X_{t}, r_{t}\right\}$ in period $t$. The forced holding cost is unavoidable and it is independent of the current decision. The second part of the marginal holding cost is an additional future holding cost (denoted by $\hat{H}_{t}$ ) incurred by producing additional (controllable) $\eta_{t}=X_{t}+q_{t}-\bar{X}_{t}$. The reason behind this split is that the forced marginal holding cost $\tilde{H}_{t}$ is fixed given production decisions in previous periods, and hence only the additional marginal holding cost $\hat{H}_{t}$ is affected by the current decision $\eta_{t}$.

Suppose that $X_{t}$ is the inventory position at the beginning of period $t$. We compute the forced holding $\operatorname{cost} \tilde{H}_{t}$ by

$$
\tilde{H}_{t}=\sum_{j=t+L}^{T+L} h_{j}\left(\left(\bar{X}_{t}-D_{[t, j]}\right)^{+}-\left(X_{t}-D_{[t, j]}\right)^{+}\right),
$$

where $h_{j}\left(\bar{X}_{t}-D_{[t, j]}\right)^{+}$is the marginal holding cost in period $j$ for producing up to $\bar{X}_{t}$ in period $t$ and $h_{j}\left(X_{t}-D_{[t, j]}\right)^{+}$is the marginal holding cost in period $j$ for producing nothing in period $t$. Similarly, the additional future holding cost $\hat{H}_{t}$ is computed as

$$
\hat{H}_{t}\left(\eta_{t}\right)=\sum_{j=t+L}^{T+L} h_{j}\left(\left(\bar{X}_{t}+\eta_{t}-D_{[t, j]}\right)^{+}-\left(\bar{X}_{t}-D_{[t, j]}\right)^{+}\right),
$$

where $h_{j}\left(\bar{X}_{t}+\eta_{t}-D_{[t, j]}\right)^{+}$stands for the marginal holding cost in period $j$ for producing an additional $\eta_{t}$ in period $t$ and $h_{j}\left(\bar{X}_{t}-D_{[t, j]}\right)^{+}$is the marginal holding cost in period $j$ for producing nothing additionally in period $t$. The backlogging cost in period $t$ is the same as the classical per-period backlogging cost, i.e.,

$$
B_{t}\left(\eta_{t}\right)=b_{t+L}\left(D_{[t, t+L]}-\left(\eta_{t}+\bar{X}_{t}\right)\right)^{+}
$$

The left graph in Figure 2 shows the split marginal costs.

Regrouping the marginal costs (Merge). After splitting the marginal costs, we next regroup them. The marginal costs fall into two categories. One is called overage cost and it includes the marginal costs incurred due to production. Specifically, the additional holding cost $\hat{H}_{t}$ is overage cost since it will increase when an additional production is made. We name the other category underage cost, which includes the marginal costs incurred due to lack of productions, such as the 
backlogging cost $B_{t}\left(\eta_{t}\right)$. For the forced holding cost $\tilde{H}_{t}$, however, it is not affected by the decision in the same period because it is pre-determined by the production made in the last period. For this reason, we compute the delayed holding cost in the subsequent period (i.e., $\tilde{H}_{t+1}$ ) as soon as the production is made in period $t$. Specifically, once the additional production quantity $\eta_{t}$ is determined, we compute the delayed holding cost by

$$
\tilde{H}_{t+1}\left(\eta_{t}\right)=\sum_{j=t+L+1}^{T+L} h_{j}\left(\left(\bar{X}_{t+1}-D_{[t, j]}\right)^{+}-\left(X_{t+1}-D_{[t, j]}\right)^{+}\right) .
$$

Note that the delayed holding cost requires to compute $r_{t+1}$ based on $f_{t}$ rather than $f_{t+1}$. Hence, the term $\bar{X}_{t+1}$ in (11) should be treated as a random variable depending on the realization of $D_{t}$.

As seen from (11), the more we produce in period $t$, the more inventory position we have at the beginning of period $t+1$ and the less delayed holding cost will be incurred. Thus, by shifting all the marginal forced holding costs to one period later, we conclude that $\tilde{H}_{t+1}$ is decreasing in $\eta_{t}$ and hence, it belongs to underage cost. The right graph in Figure 2 illustrates the shifted cost.

Figure 2 Marginal costs under the SMB policy

SMB policy (Balance). We describe the SMB policy as follows: At the beginning of each period $t$, we first calculate the balancing quantity $\eta_{t}^{S M B}$, which balances the conditional expected overage cost against the conditional expected underage cost. In other words, $\eta_{t}^{S M B}$ solves

$$
\mathbb{E}\left[\hat{H}_{t}\left(\eta_{t}\right) \mid f_{t}\right]=\mathbb{E}\left[\tilde{H}_{t+1}\left(\eta_{t}\right)+B_{t}\left(\eta_{t}\right) \mid f_{t}\right]
$$

Then the SMB policy produces $q_{t}^{S M B}=\eta_{t}^{S M B}+\bar{x}_{t}-x_{t}$ in period $t$ when $X_{t}=x_{t}$ is realized. Here in the SMB policy, the balancing quantity must exist due to the following facts: (i) $\hat{H}_{t}\left(\eta_{t}\right), \tilde{H}_{t+1}\left(\eta_{t}\right)$ and $B_{t}\left(\eta_{t}\right)$ are continuous; (ii) $\hat{H}_{t}\left(\eta_{t}\right)$ is non-decreasing in $\eta_{t}$ while $\tilde{H}_{t+1}\left(\eta_{t}\right)+B_{t}\left(\eta_{t}\right)$ are nonincreasing in $\eta_{t}$; (iii) As $\eta_{t}$ increases from 0 to $+\infty$, the LHS of (12) also increases from 0 to $+\infty$ while the RHS of (12) decreases from a positive number to 0. Moreover, the balancing quantity $\eta_{t}^{S M B}$ can be computed efficiently using a bisection search method.

To evaluate the total cost of a given policy $P$ in a convenient way, we define the required service level in period $T+L+1$ to be zero, i.e., $r_{T+1}=0$. Under this convention, the forced costs must vanish in period $t+1$, i.e., $\tilde{H}_{T+1}^{P}=0$. Then for a given policy $P$, the total $\operatorname{cost} \mathcal{C}(P)$ is given by

$$
\mathcal{C}(P)=\sum_{t=1}^{T}\left(\left(\tilde{H}_{t}^{P}+\hat{H}_{t}^{P}\right)+B_{t}^{P}\right)=\sum_{t=1}^{T}\left(\hat{H}_{t}^{P}+\left(\tilde{H}_{t+1}^{P}+B_{t}^{P}\right)\right)+\tilde{H}_{1}^{P} .
$$


We note that the delayed holding cost $\tilde{H}_{t+1}^{P}$ for any policy $P$ is computed using the demand information $f_{t}$ obtained in the previous period. Also, note that the forced cost $\tilde{H}_{1}^{P}$ can be calculated without knowing specific stochastic demand information and the policy $P$ we use. Hence, they are realized at the beginning of the planning horizon and are fixed in any policy $P$ we refer to.

\subsection{Worst-Case Analysis of the SMB Policy}

Now we establish the worst-case guarantee of 2 for the proposed SMB policy, which is one of the main results in this paper. Due to service level constraints, the forced holding cost and additional holding cost components need to be considered separately. To this end, we use an algebraic method to prove our desired results, which departs from the unit-matching techniques used in Levi et al. (2007).

To begin with, we define the following random sets of periods:

- $\mathcal{T}_{H}=\left\{t \mid Y_{t}^{S M B}<Y_{t}^{O P T}\right\}$ denotes the set of periods in which the optimal policy yields more ending inventory in period $t+L$ than the SMB policy;

- $\mathcal{T}_{B}=\left\{t \mid Y_{t}^{S M B} \geq Y_{t}^{O P T}\right\}$ denotes the set of periods in which the optimal policy yields less or equal ending inventory in period $t+L$ compared to the SMB policy; it is evident that $\mathcal{T}_{H}$ and $\mathcal{T}_{B}$ are disjoint sets and $\mathcal{T}_{H} \cup \mathcal{T}_{B}=\{1,2, \ldots, T\}$.

Our result is based on the following lemmas and the detailed proofs can be found in Appendix A. The key idea is to calculate the total cost of the SMB policy using periods in sets $\mathcal{T}_{H}$ and $\mathcal{T}_{B}$. Then in each period, we bound either the overage cost or the underage cost by the corresponding cost for the optimal policy, according to which set the current period belongs to.

LeMma 1. $\mathbb{E}[\mathcal{C}(S M B)]=2 \mathbb{E}\left[\sum_{t \in \mathcal{T}_{H}} \hat{H}_{t}^{S M B}+\sum_{t \in \mathcal{T}_{B}}\left(\tilde{H}_{t+1}^{S M B}+B_{t}^{S M B}\right)\right]+\tilde{H}_{1}^{S M B}$.

First, we consider the case when $t \in \mathcal{T}_{B}$. In this case, the ending inventory position in period $t$ for the optimal policy is lower than that of the SMB policy, and therefore it yields more backlogging cost in the current period. Moreover, given the relatively lower ending inventory position for the optimal policy, the inventory position at the beginning of the next period for the optimal policy must also be lower. Thus, the optimal policy must yield a larger forced holding cost in period $t+1$. We summarize these observations in the following lemma.

Lemma 2. For any $t \in \mathcal{T}_{B}$, we have:

1. $B_{t}^{S M B} \leq B_{t}^{O P T}$;

2. $\tilde{H}_{t+1}^{S M B} \leq \tilde{H}_{t+1}^{O P T}$. 
For any period $t \in \mathcal{T}_{H}$, the ending inventory of the SMB policy is lower than that of the optimal policy. Consider consecutive periods $\left[t^{1}, t^{2}\right] \subseteq \mathcal{T}_{H}$. At the beginning of period $t^{1}$, the inventory position of the SMB policy is higher while at the end of period $t^{2}$ the inventory of the SMB policy is lower. This implies that the SMB policy must make fewer additional productions than the optimal policy. As a result, the additional holding cost of the SMB policy is dominated by the additional holding cost of the optimal policy. We summarize this result in the following lemma:

LEMMA 3. For $t \in \mathcal{T}_{H}, \sum_{t \in \mathcal{T}_{H}} \hat{H}_{t}^{S M B} \leq \sum_{t \in \mathcal{T}_{H}} \hat{H}_{t}^{O P T}$.

Combining Lemma 1 to Lemma 3 together, we have

$$
\begin{aligned}
\mathbb{E}[\mathcal{C}(S M B)] & =2 \mathbb{E}\left[\sum_{t \in \mathcal{T}_{H}} \hat{H}_{t}^{S M B}+\sum_{t \in \mathcal{T}_{B}}\left(\tilde{H}_{t+1}^{S M B}+B_{t}^{S M B}\right)\right]+\tilde{H}_{1}^{S M B} \\
& \leq 2 \mathbb{E}\left[\sum_{t \in \mathcal{T}_{H}} \hat{H}_{t}^{O P T}+\sum_{t \in \mathcal{T}_{B}}\left(\tilde{H}_{t+1}^{O P T}+B_{t}^{O P T}\right)\right]+\tilde{H}_{1}^{O P T} \\
& \leq 2 \mathbb{E}\left[\sum_{t=1}^{T}\left(\hat{H}_{t}^{O P T}+\tilde{H}_{t+1}^{O P T}+B_{t}^{O P T}\right)+\tilde{H}_{1}^{O P T}\right]=2 \mathbb{E}[\mathcal{C}(O P T)] .
\end{aligned}
$$

Hence, we have proved the following theorem, which provides a worst-case performance guarantee on the result of the SMB policy.

THEOREM 1. The SMB policy has a worst-case performance guarantee of two, i.e., for each instance of the backlogging model under service level constraints, the expected cost of the $S M B$ policy is at most twice the expected cost of an optimal solution, i.e., $\mathbb{E}[\mathcal{C}(S M B)] \leq 2 \mathbb{E}[\mathcal{C}(O P T)]$.

\section{Remanufacturing System with Service Level Requirements}

We consider a hybrid manufacturing/remanufacturing inventory system that produces a single product over a planning horizon of $T$ periods, indexed by $t=1, \ldots, T$. In each period $t$, the manufacturer receives random customer demand $D_{t}$ for serviceable products and random product return $U_{t}$. A serviceable product can be either manufactured from raw materials or remanufactured from a returned product (also known as a core). At the end of each period, leftover returned products and unsold serviecable products are carried to the next period, and unsatisfied demand units are backlogged to the next period. Our objective is to coordinate a sequence of manufacturing and remanufacturing quantities over the planning horizon so as to minimize the expected total cost.

We assume that demand is indifferent between manufacturing and remanufacturing products. For example, functional parts from the returned products can be used in producing new products (which helps reduce the manufacturing cost). The difference between a new and a remanufactured product is whether some components are new or recovered from cores (see Zhou et al. (2011), 
Gong and Chao (2013), Tao and Zhou (2014) for more examples). Following Simpson (1978), we assume that manufacturing and remanufacturing operations have identical lead times $L$. For nonidentical lead times, one may derive similar heuristic algorithms, but the approximation results will not hold even without service level constraints (see $\S 5.1$ in Tao and Zhou (2014) for a more detailed discussion on non-identical lead times).

The major difference between the remanufacturing model and the basic model lies in the dual modes of production, i.e., the manufacturer can either produce by remanufacturing a returned product or by regular way using raw materials. This leads to the nonlinear production cost, which makes the model challenging. In the following, we will first formulate our model using the DP approach and show the structure of optimal policies. Then we will generalize the proposed SMB policy to solve the remanufacturing model, which also guarantees us a worst-case performance of two. Our technique is based on Tao and Zhou (2014), in which they proposed a two approximation algorithm for the remanufacturing system without service level requirement. However, our results are different in the following ways. First, due to the service level requirements presented in our model, our algorithm (see §4.2) departs from the one proposed in Tao and Zhou (2014). Second, in the worst-case analysis, amortizing production costs of the modified SMB policy is different since we need to handle two parts of split production cost, i.e., the forced production cost and the additional production cost (see $\S 4.3$ for more details).

\subsection{System Dynamics and DP Formulation}

Here we adopt most notation described in $\S 2$. In remanufacturing model, we also consider a $T$ period inventory control problem. The sequence of events in each period $t$ is as follows. First, the manufacturer reviews the starting inventory levels of serviceable products and returned products. After observing the information set $f_{t}$ (defined below), the manufacturer decides the remanufacturing quantity $q_{t}^{1}$ and the manufacturing quantity $q_{t}^{2}$. Both production methods have the same lead times $L$ (see, e.g., Zhou et al. (2011)). The total number of productions at period $t$ is computed by $q_{t}=q_{t}^{1}+q_{t}^{2}$. We use $c_{t}^{1}$ and $c_{t}^{2}$ to denote the remanufacturing cost and the manufacturing cost with $c_{t}^{1}<c_{t}^{2}$, since it is usually cheaper to remanufacture. Following discussion from Zhou et al. (2011), we also assume that $c_{t}^{2}-c_{t}^{1}$ is non-increasing. This assumption holds in practice where manufacturing costs can be reduced significantly over time while remanufacturing costs are lower and hard to be reduced. Then the random customer demand $D_{t}=d_{t}$ and the random product return $U_{t}=u_{t}$ are realized. Finally, all costs (including manufacturing, remanufacturing, holding, and backlogging costs) for this period are assessed. Note that we use $h_{t}^{1}$ to denote the unit holding 
cost for each returned product and $h_{t}^{2}$ to denote the unit holding cost for each serviceable product. The unit backlogging cost is denoted by $b_{t}$.

In the remanufacturing model, the information set $f_{t}$ is realized at the beginning of period $t$, which consists of the realized demands $\left(d_{1}, \ldots, d_{t-1}\right)$, the realized returns $\left(u_{1}, \ldots, u_{t-1}\right)$ and some exogenous information $\left(\rho_{1}, \ldots, \rho_{t}\right)$ such as the state of economy. The conditional joint distribution of future demand and returns $\left(D_{t}, \ldots, D_{T}, U_{t}, \ldots, U_{T}\right)$ is determined by the information set $f_{t}$.

We note that the return process is assumed to be exogenous, which is a predominant assumption in this line of literature (see Zhou et al. (2011), Gong and Chao (2013), Tao and Zhou (2014) among others). However, one may argue that the returns should be capped by past sales, which essentially become endogenous. Unfortunately, the methods of this paper (and also in the literature) will not be able to handle such a case. Nevertheless, the current model can be thought of as a random capacity model with two suppliers, in which the equivalence is described in Appendix C.

Identical to the basic backlogging model, we consider either $\alpha$ or $\beta$ type of service level constraint in the remanufacturing model. As already discussed in $\S 2$, both $\alpha$ and $\beta$ service level constraints can be simplified to a lower bound $r_{t}\left(f_{t}\right)$ on the inventory position after ordering at period $t$. The lower bound $r_{t}$ can be readily computed using numerical methods discussed in $\S 2$.

Before formally formulating the problem, we can first simplify the problem via cost transformation. Let $x_{t}$ be the inventory position at the beginning of period $t$ and $w_{t}$ be the total number of returned products at the beginning of period $t$. Then we have

$$
\begin{aligned}
& x_{t+1}=x_{t}+q_{t}-d_{t}, \quad \forall t=1, \ldots, T-1, \\
& w_{t+1}=w_{t}-q_{t}^{1}+u_{t}, \quad \forall t=1, \ldots, T-1 .
\end{aligned}
$$

Let $\Pi$ be the set of all feasible policies and any policy $\pi \in \Pi$ is a mapping from $\left(x_{t}, w_{t}, r_{t}, f_{t}\right)$ to $\left(q_{t}^{1}, q_{t}^{2}\right) \in \mathcal{Q}\left(x_{t}, w_{t}, r_{t}\right)$, where $\mathcal{Q}\left(x_{t}, w_{t}, r_{t}\right)=\left\{\left(q_{t}^{1}, q_{t}^{2}\right) \mid 0 \leq q_{t}^{1} \leq w_{t}, q_{t}^{2} \geq 0, q_{t}^{1}+q_{t}^{2} \geq r_{t}-x_{t}\right\}$ is the feasible set of the production quantities for two production methods. Since the evolution of $F_{n}$ is assumed independent of the policy $\pi$, the total expected cost for policy $\pi$ is computed as

$$
\begin{aligned}
& C^{\pi}=\mathbb{E}\left[\sum _ { t = 1 } ^ { T } \left(h_{t+L}^{2}\left(X_{t}^{\pi}+q_{t}^{\pi}-D_{[t, t+L]}\right)^{+}+h_{t}^{1}\left(W_{t}^{\pi}-q_{t}^{1, \pi}+U_{t}\right)\right.\right. \\
& \left.\left.+b_{t+L}\left(D_{[t, t+L]}-X_{t}^{\pi}-q_{t}^{\pi}\right)^{+}+c_{t}^{1} q_{t}^{1, \pi}+c_{t}^{2} q_{t}^{2, \pi}\right) \mid f_{1}\right] .
\end{aligned}
$$

Using (15), we can rewrite $W_{t}^{\pi}=w_{1}+\sum_{i=1}^{t-1} U_{i}-\sum_{i=1}^{t-1} q_{i}^{1, \pi}$. Thus, if we define $\delta_{t}^{h}=\sum_{i=t}^{T} h_{t}^{1}$, then $\mathbb{E}\left[\sum_{t=1}^{T} h_{t}^{1}\left(W_{t}^{\pi}-q_{t}^{1, \pi}+U_{t}\right) \mid f_{1}\right]=\delta_{1}^{h} w_{1}+\mathbb{E}\left[\sum_{t=1}^{T} \delta_{t}^{h} U_{t} \mid f_{1}\right]-\sum_{t=1}^{T} \delta_{t}^{h} q_{t}^{1, \pi}$. Hence, we can simplify the total expected cost as

$$
C^{\pi}=\mathbb{E}\left[\sum_{t=1}^{T}\left(h_{t+L}^{2}\left(X_{t}^{\pi}+q_{t}^{\pi}-D_{[t, t+L]}\right)^{+}+b_{t+L}\left(D_{[t, t+L]}-X_{t}^{\pi}-q_{t}^{\pi}\right)^{+}+\left(c_{t}^{1}-\delta_{t}^{h}\right) q_{t}^{1, \pi}+c_{t}^{2} q_{t}^{2, \pi}\right) \mid f_{1}\right]
$$




$$
\begin{gathered}
+\delta_{1}^{h} w_{1}+\mathbb{E}\left[\sum_{t=1}^{T} \delta_{t}^{h} U_{t} \mid f_{1}\right] \\
=\sum_{t=1}^{T} \mathbb{E}\left[\bar{h}_{t+L}\left(x_{t}+q_{t}-D_{[t, t+L]}\right)^{+}+\bar{b}_{t+L}\left(D_{[t, t+L]}-x_{t}-q_{t}\right)^{+}+\bar{c}_{t} q_{t}^{2} \mid f_{1}\right]+C_{0},
\end{gathered}
$$

where

$$
\begin{aligned}
& \bar{h}_{t+L}=h_{t+L}^{2}+c_{t}^{1}-c_{t+1}^{1}-h_{t}^{1}, \quad \bar{b}_{t+L}=b_{t+L}-c_{t}^{1}+c_{t+1}^{1}+h_{t}^{1}, \quad \bar{c}_{t}=c_{t}^{2}-c_{t}^{1}+\delta_{t}^{h}, \\
& C_{0}=\delta_{1}^{h} w_{1}+\mathbb{E}\left[\sum_{t=1}^{T} \delta_{t}^{h} U_{t} \mid f_{1}\right]+\mathbb{E}\left[\sum_{t=1}^{T}\left(c_{t}^{1}-\delta_{t}^{h}\right) D_{t+L} \mid f_{1}\right] .
\end{aligned}
$$

Note that in the above equation, $C_{0}$ is a uncontrollable cost (independent of any feasible policies), and $\bar{h}_{t+L}, \bar{b}_{t+L}$ and $\bar{c}_{t}$ are the modified unit holding cost, the unit backlogging cost and the unit manufacturing cost, respectively. Therefore, we can transform the per-unit costs so that any remanufacturing system is equivalent to another remanufacturing system with zero holding cost on returned products and zero remanufacturing cost, up to a constant difference between their expected costs. We assume $\bar{h}_{t+L} \geq 0$ and $\bar{b}_{t+L} \geq 0$ for all $t=1,2, \ldots, T$, to prevent speculation for holding inventory or backorders. We also have $\bar{c}_{t}>0$ and non-increasing since $c_{t}^{2}>c_{t}^{1}$ and $c_{t}^{2}-c_{t}^{1}$ is non-increasing. Thus, in the subsequent discussion, we will focus on the system with zero holding cost on returned products and zero remanufacturing cost, with positive inventory holding cost, positive backlogging cost and positive, non-increasing manufacturing cost.

To derive a DP formulation, we first describe the state vector as follows. It consists of a time period $t$, inventory position $x_{t}$ at the beginning of period $t$, total number of returned products $w_{t}$ at the beginning of period $t$, and information $f_{t} \in \mathcal{F}_{t}$. The system dynamics are given by (14) and (15) with initial inventory position $x_{1}$ and initial number of available returned products $w_{1}$. The value function $v_{t}\left(x_{t}, w_{t}, f_{t}\right)$ is the minimal expected cost from period $t+L$ to period $T+L$. In each period $t$, given the state vector $\left(x_{t}, w_{t}, f_{t}\right)$, we need to decide the remanufacturing quantity $q_{t}^{1}$ and the manufacturing quantity $q_{t}^{2}$. The remanufacturing quantity $q_{t}^{1}$ is bounded above by $w_{t}$, while the servicelevel constraint enforces $y_{t}=x_{t}+q_{t}^{1}+q_{t}^{2}$ bounded below by $r_{t}$. Hence, feasible choices of the two types of quantities are in the set

$$
\mathcal{Q}\left(x_{t}, w_{t}, r_{t}\right)=\left\{\left(q_{t}^{1}, q_{t}^{2}\right) \mid 0 \leq q_{t}^{1} \leq w_{t}, q_{t}^{2} \geq 0, q_{t}^{1}+q_{t}^{2} \geq r_{t}-x_{t}\right\}
$$

Using the transformed unit costs described above, we can write the Bellman's equations as

$$
\begin{aligned}
& v_{T+1}\left(x_{T+1}, w_{T+1}, f_{T+1}\right)=0, \quad \forall x_{T+1} \in \mathbb{R}, w_{T+1} \in \mathbb{R}^{+} \cup\{0\}, f_{T+1} \in \mathcal{F}_{T+1}, \\
& v_{t}\left(x_{t}, w_{t}, f_{t}\right)=\min _{q_{t}^{1}, q_{t}^{2} \in \mathcal{Q}\left(x_{t}, w_{t}, r_{t}\right)}\left\{\bar{G}_{t}\left(y_{t}, f_{t}\right)+\bar{c}_{t} q_{t}^{2}+\mathbb{E}\left[v_{t+1}\left(y_{t}-D_{t}, w_{t}-q_{t}^{1}+U_{t}, \mathcal{F}_{t+1}\right) \mid f_{t}\right]\right\}, \\
& \forall t=1, \ldots, T,
\end{aligned}
$$

\section{This article is protected by copyright. All rights reserved}


where $\bar{G}_{t}\left(y_{t}, f_{t}\right)=\mathbb{E}\left[\bar{h}_{t+L}\left(y_{t}-D_{[t, t+L]}\right)^{+}+\bar{b}_{t+L}\left(D_{[t, t+L]}-y_{t}\right)^{+}\right]$. Using the above DP formulation (16), the structure of optimal policies is characterized in the following proposition. A detailed proof is given in Appendix B.

Proposition 2. For the inventory control problem defined in (16), an optimal policy is a total base-stock (including both manufacturing and remanufacturing) policy. More specifically, there exists $\left\{s\left(w_{t}, f_{t}\right)\right\}_{t=1}^{T}$ such that

$$
y_{t}^{*}\left(x_{t}, w_{t}\right)= \begin{cases}\max \left\{r_{t}, s\left(w_{t}, f_{t}\right)\right\}, & \text { if } x_{t}<s\left(w_{t}, f_{t}\right) \\ \max \left\{r_{t}, x_{t}\right\}, & \text { if } x_{t} \geq s\left(w_{t}, f_{t}\right) .\end{cases}
$$

and

$$
q_{t}^{1^{*}}\left(x_{t}, w_{t}\right)=\min \left\{w_{t}, y_{t}^{*}-x_{t}\right\}, q_{t}^{2^{*}}\left(x_{t}, w_{t}\right)=y_{t}^{*}-x_{t}-q_{t}^{1^{*}}\left(x_{t}\right)
$$

Proposition 2 asserts that any optimal policy has the following structure: if the inventory position in period $t$ is no less than the threshold $s\left(w_{t}, f_{t}\right)$, an optimal policy produces up to the required service level $r_{t}$; otherwise, it brings the total inventory position (after production) to $\max \left\{r_{t}, s\left(w_{t}, f_{t}\right)\right\}$. Therefore, the higher the service level, the more orders are placed by the optimal policy. Moreover, the optimal policy will remanufacture returned products as many as possible before manufacturing new products.

\subsection{Modified Split-Merge-Balance Policy}

The key idea of modified SMB policy (MSMB for short) is similar to the SMB policy proposed in $\S 3.2$, which has three phases, namely, split, merge and balance. However, in the remanufacturing system, we have to consider production cost in addition to the holding and backlogging costs. Recall that $\bar{X}_{t}=\max \left\{X_{t}, r_{t}\right\}$ is the required service level, $\eta_{t}=y_{t}-\bar{X}_{t}$ is the controllable producing quantity in period $t$, and the marginal production cost in period $t$ is given by

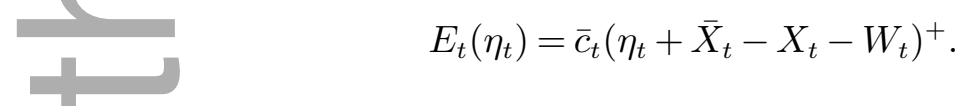

First, we use the same definitions of the forced holding cost $\tilde{H}_{t}$, the future holding cost $\hat{H}_{t}$ and the backlogging cost $B_{t}$ as defined in (8)-(10). Similar as splitting the holding cost in $\S 3.2$, we also split the marginal production cost into two parts: the forced production cost (denoted by $\tilde{E}_{t}$ ) which accounts for the cost of producing up to the required service level $\bar{X}_{t}=\max \left\{X_{t}, r_{t}\right\}$ in period $t$ and the additional production cost (denoted by $\hat{E}_{t}$ ) determined by the amount of additional (controllable) produces $\eta_{t}$. Specifically, if the number of returned products in period $t$ is denoted by $W_{t}$, the forced production cost $\tilde{E}_{t}$ is computed by

$$
\tilde{E}_{t}=\bar{c}_{t}\left(\bar{X}_{t}-X_{t}-W_{t}\right)^{+}
$$


and the additional production cost is

$$
\hat{E}_{t}\left(\eta_{t}\right)=\bar{c}_{t}\left(\left(\eta_{t}+\bar{X}_{t}-X_{t}-W_{t}\right)^{+}-\left(\bar{X}_{t}-X_{t}-W_{t}\right)^{+}\right)
$$

Next, we regroup the costs based on whether they are overage cost or underage cost. Similar as in the manufacturing model, we shift the forced holding cost $\tilde{H}_{t}$ to one period later, as defined in (11), which belongs to underage cost. For production costs, it is evident that the additional production cost $\hat{E}_{t}\left(\eta_{t}\right)$ is overage cost since it increases when $\eta_{t}$ increases (i.e., more productions are made). For the forced production cost $\tilde{E}_{t}$, although it does not depend on the decision $\eta_{t}$ in the current period, it occurs due to the lack of production in the previous period. Hence, by shifting the cost to one period later, we conclude that

$$
\tilde{E}_{t+1}\left(\eta_{t}\right)=\bar{c}_{t+1}\left(\bar{X}_{t+1}-X_{t+1}-W_{t+1}\right)^{+}
$$

is non-increasing in $\eta_{t}$ and it belongs to underage cost. Similar as the delayed holding cost, the delayed production cost requires to compute $r_{t+1}$ based on $f_{t}$ rather than $f_{t+1}$. Hence, the term $\bar{X}_{t+1}$ in (19) should be treated as a random variable depending on the realization of $D_{t}$.

To summarize, if we use $\Phi_{t}$ and $\Psi_{t}$ to denote the total overage cost and the total underage cost in period $t$, we have $\Phi_{t}\left(\eta_{t}\right)=\hat{H}_{t}\left(\eta_{t}\right)+\hat{E}_{t}\left(\eta_{t}\right)$ and $\Psi_{t}\left(\eta_{t}\right)=\tilde{H}_{t+1}\left(\eta_{t}\right)+\tilde{E}_{t+1}\left(\eta_{t}\right)+B_{t}\left(\eta_{t}\right)$. Figure 3 illustrates the split phase and the merge phase of the MSMB policy.

Figure 3 Marginal costs under the SMB policy

Finally, we balance the overage cost against the underage cost, i.e., $\eta_{t}^{M S M B}$ solves

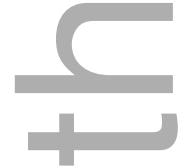

$$
\mathbb{E}\left[\Phi_{t}\left(\eta_{t}\right) \mid f_{t}\right]=\mathbb{E}\left[\Psi_{t}\left(\eta_{t}\right) \mid f_{t}\right]
$$

Thus, the MSMB policy produces $q_{t}^{M S M B}=\eta_{t}^{M S M B}+\bar{x}_{t}-x_{t}$ in period $t$. Moreover, it fully utilizes the returned products to remanufacture, i.e., $q_{t}^{1, M S M B}=\min \left\{w_{t}, q_{t}^{M S M B}\right\}$ and $q_{t}^{2, M S M B}=\left(q_{t}^{M S M B}-\right.$ $\left.w_{t}\right)^{+}$.

\subsection{Worst-Case Analysis of the MSMB Policy}

Now we establish the worst-case guarantee of two for the proposed MSMB policy. The main difficulty in our analysis is to amortize the production costs of the MSMB policy against that of the optimal policy. Our proof techniques are based on Tao and Zhou (2014), in which the authors 
constructed a set of periods such that the total production costs of the balancing policy are dominated by that of the optimal policy. They further showed the same inequality holds for the set of periods in which the optimal policy yields less or equal inventory compared to the balancing policy (see Lemma 4 of Tao and Zhou (2014)). However, our proof is different since we need to bound both the forced production cost and the additional production cost at the same time. As a result, the same inequality does not hold any more; instead, we delay the forced production cost to one period later and bound the total additional production costs in periods $\mathcal{T}_{\Phi}$ plus the total delayed production costs in periods $\mathcal{T}_{\Psi}$, which is crucial in our analysis (see Lemma 6).

In the following discussion, we will only focus on a particular type of policy, namely the rational policies. These policies will not manufacture at a higher per-unit cost unless there are no returned products to remanufacture. As we have already shown in Proposition 2, any optimal policy (indicated by OPT) is a rational policy and the MSMB policy described above is also rational. We will use superscripts to specify which policy we refer to.

We define new variables and introduce new notation. For any given policy $P$, let $W_{t}^{P}$ be the total number of returned products in period $t$, and $S_{t}^{P}$ be the remaining number of returned products after producing up to the service level $\bar{X}_{t}^{P}$ in period $t$. We also split the additional production quantity $\eta_{t}$ into two parts, denoted by $\eta_{t}^{1, P}$ and $\eta_{t}^{2, P}$, representing the additional remanufacturing quantity and additional manufaturing quantity of a given policy $P$. Because we only consider rational policies, we must have $\eta_{t}^{1, P} \leq S_{t}^{P}$ and $\eta_{t}^{2, P}=\left(\eta_{t}^{P}-S_{t}^{P}\right)^{+}$. Moreover, the system dynamics follow

$$
\left\{\begin{array}{l}
W_{t}^{P}=S_{t-1}^{P}-\eta_{t-1}^{1, P}+U_{t-1} \\
S_{t}^{P}=\left(W_{t}^{P}-\left(\bar{X}_{t}^{P}-X_{t}^{P}\right)\right)^{+} \\
X_{t}^{P}=\bar{X}_{t-1}^{P}+\eta_{t-1}^{1, P}+\eta_{t-1}^{2, P}-d_{t-1}
\end{array}\right.
$$

We rewrite the additional production cost as $\hat{E}_{t}^{P}=\bar{c}_{t} \eta_{t}^{2, P}$, and define three random sets of periods as follows.

- $\mathcal{T}_{\Phi}=\left\{t \mid Y_{t}^{M S M B}<Y_{t}^{O P T}\right\}$ denotes the set of periods $t$ in which the optimal policy yields more ending inventory in periods $t+L$ than the MSMB policy;

- $\mathcal{T}_{\Psi}=\left\{t \mid Y_{t}^{M S M B} \geq Y_{t}^{O P T}\right\}$ denotes the set of periods $t$ in which the optimal policy yields less or equal ending inventory in period $t+L$ compared to the MSMB policy; it is evident that $\mathcal{T}_{\Phi}$ and $\mathcal{T}_{\Psi}$ are disjoint sets and $\mathcal{T}_{\Phi} \cup \mathcal{T}_{\Psi}=\{1,2, \ldots, T\}$

- $\mathcal{T}_{c}=\left\{t \mid \bar{X}_{t}^{M S M B}+S_{t}^{M S M B}+\eta_{t}^{2, M S M B} \geq \bar{X}_{t}^{O P T}+S_{t}^{O P T}+\eta_{t}^{2, O P T}\right\}$. Following the system dynamics in (21), we can equivalently present the set as $\mathcal{T}_{c}=\left\{t \mid W_{t+1}^{M S M B}+X_{t+1}^{M S M B} \geq W_{t+1}^{O P T}+X_{t+1}^{O P T}\right\}$. The quantity $W_{t}^{P}+X_{t}^{P}$ stands for the maximum producing-up-to level without having any production cost in period $t$ (which we refer to as the free-production level). The set $\mathcal{T}_{c}$ can be interpreted 
as the periods in which the free-production level in the next period for the MSMB policy is lower than that of the optimal policy.

Our main results are based on the following lemmas.

LEMMA 4. $\mathbb{E}[\mathcal{C}(M S M B)]=2 \mathbb{E}\left[\sum_{t \in \mathcal{T}_{\Phi}} \Phi_{t}^{M S M B}+\sum_{t \in \mathcal{T}_{\Psi}} \Psi_{t}^{M S M B}\right]+\tilde{H}_{1}^{M S M B}+\tilde{E}_{1}^{M S M B}$.

We note that the both forced holding cost $\tilde{H}_{1}^{M S M B}$ and forced production cost $\tilde{E}_{1}^{M S M B}$ can be computed without knowing specific stochastic demand information and the policy we use. Hence, they are realized at the beginning of the planning horizon and are fixed in any policy $P$ we refer to.

The following lemma restates the results proved in Lemmas 2 and 3, under the modified SMB policy. The proof is identical to the proofs of Lemmas 2 and 3, and thus omitted here.

LEMMA 5. 1. For any $t \in \mathcal{T}_{\Psi}$, we have $B_{t}^{M S M B} \leq B_{t}^{O P T}$ and $\tilde{H}_{t+1}^{M S M B} \leq \tilde{H}_{t+1}^{O P T}$.

2. For $t \in \mathcal{T}_{\Phi}$, we have $\sum_{t \in \mathcal{T}_{\Phi}} \hat{H}_{t}^{M S M B} \leq \sum_{t \in \mathcal{T}_{\Phi}} \hat{H}_{t}^{O P T}$.

The next lemma is crucial in our analysis, which deals with production costs. The difficulty of the analysis lies in the fact that the production cost does not only depend on the ending inventory level but also depends on the number of returned products $W_{t}$. For this reason, we first compare the production cost of the MSMB policy and that of the optimal policy for sets $\mathcal{T}_{c}$ and $\mathcal{T}_{c}^{c}$. When $t \in \mathcal{T}_{c}$, the free-production level for the MSMB policy is higher than that of the optimal policy in the next period $t+1$. Therefore, the forced production cost $\tilde{E}_{t+1}$ for the MSMB policy must be lower. For set $\mathcal{T}_{c}^{c}$, consider any consecutive time interval $\left[t_{1}, t_{2}\right]$ that belongs to $\mathcal{T}_{c}^{c}$. Compared with the optimal policy, the free-production level for the MSMB policy is higher at the beginning of period $t_{1}$, while it becomes lower at the end of period $t_{2}$. This can happen only when the MSMB policy uses more free productions. As a result, the total production cost for the MSMB policy during this time interval must be less than that of the optimal policy. Finally, we extend the results to sets $\mathcal{T}_{\Phi}$ and $\mathcal{T}_{\Psi}$ using the fact that $\eta_{t}^{2, M S M B}=0$ for all $t \in \mathcal{T}_{\Phi} \cap \mathcal{T}_{c}$ and $\eta_{t}^{2, O P T}=0$ for all $t \in \mathcal{T}_{\Psi} \cap \mathcal{T}_{c}^{c}$.

Connection and comparison with Tao and Zhou (2014). Our construction of the set $\mathcal{T}_{c}$ is based on the technique used in Tao and Zhou (2014), but the analysis is different in the following aspects. First, they showed that the total production cost of the balancing policy in periods $\mathcal{T}_{c}$ is no more than that of the optimal policy, i.e.,

$$
\sum_{t \in \mathcal{T}_{c}^{c}}\left(\hat{E}_{t}^{M S M B}+\tilde{E}_{t}^{M S M B}\right) \leq \sum_{t \in \mathcal{T}_{c}^{c}}\left(\hat{E}_{t}^{O P T}+\tilde{E}_{t}^{O P T}\right) .
$$

However, the above inequality does not hold in our model since the forced production $\operatorname{cost} \tilde{E}_{t}^{M S M B}$ is pre-determined by the previous decisions. This motivates us to consider a delayed production 
cost which shifts the forced production cost to one period later. The reason behind this is that the delayed production cost is determined as soon as the productions are made in the current period and it can be treated as a penalty for not producing enough in the current period. We show that the total additional production cost plus the total delayed production cost of our MSMB policy in periods $\mathcal{T}_{c}$ are no more than those of the optimal policy. (See the second inequality in Lemma 6).

Secondly, after comparing the total production costs in periods $\mathcal{T}_{c}$, Tao and Zhou (2014) proved the same inequality holds in periods $\mathcal{T}_{\Phi}$ (see Lemma 4 in Tao and Zhou (2014)), i.e.,

$$
\sum_{t \in \mathcal{T}_{\Phi}}\left(\hat{E}_{t}^{M S M B}+\tilde{E}_{t+1}^{M S M B}\right) \leq \sum_{t \in \mathcal{T}_{\Phi}}\left(\hat{E}_{t}^{O P T}+\tilde{E}_{t+1}^{O P T}\right) .
$$

Again, this inequality does not hold in our case; instead, we show that the total additional cost in periods $\mathcal{T}_{\Phi}$ plus the total delayed production cost in periods $\mathcal{T}_{\Psi}$ are dominated by those of the optimal policy (see the third inequality in Lemma 6). The idea is to bound the overage cost in periods $\mathcal{T}_{\Phi}$ and the underage cost in periods $\mathcal{T}_{\Psi}$. We summarize our results in the following lemma.

LEMMA 6. For the production costs, we have

1. For $t \in \mathcal{T}_{c}, \tilde{E}_{t+1}^{M S M B} \leq \tilde{E}_{t+1}^{O P T} ;$ For $t \in \mathcal{T}_{c}^{c}, \tilde{E}_{t+1}^{M S M B} \geq \tilde{E}_{t+1}^{O P T}$;

2. $\sum_{t \in \mathcal{T}_{c}^{c}}\left(\hat{E}_{t}^{M S M B}+\tilde{E}_{t+1}^{M S M B}\right) \leq \sum_{t \in \mathcal{T}_{c}^{c}}\left(\hat{E}_{t}^{O P T}+\tilde{E}_{t+1}^{O P T}\right)$;

3. $\sum_{t \in \mathcal{T}_{\Phi}} \hat{E}_{t}^{M S S M B}+\sum_{t \in \mathcal{T}_{\Psi}} \tilde{E}_{t+1}^{M S M B} \leq \sum_{t \in \mathcal{T}_{\Phi}} \hat{E}_{t}^{O P T}+\sum_{t \in \mathcal{T}_{\Psi}} \tilde{E}_{t+1}^{O P T}$.

Combining Lemmas 4, 5, and 6, we have

$$
\begin{array}{rl}
\mathbb{E}[\mathcal{C}(M S M B)]= & 2 \mathbb{E}\left[\sum_{t \in \mathcal{T}_{\Phi}} \Phi_{t}^{M S M B}+\sum_{t \in \mathcal{T}_{\Psi}} \Psi_{t}^{M S M B}\right]+\tilde{H}_{1}^{M S M B}+\tilde{E}_{1}^{M S M B} \\
= & 2 \mathbb{E}\left[\sum_{t \in \mathcal{T}_{\Phi}} \hat{H}_{t}^{M S M B}+\sum_{t \in \mathcal{T}_{\Psi}}\left(\tilde{H}_{t+1}^{M S M B}+B_{t}^{M S M B}\right)+\left(\sum_{t \in \mathcal{T}_{\Phi}} \hat{E}_{t}^{M S M B}+\sum_{t \in \mathcal{T}_{\Psi}} \tilde{E}_{t+1}^{M S M B}\right)\right] \\
& +\tilde{H}_{1}^{M S M B}+\tilde{E}_{1}^{M S M B} \\
\leq & 2 \mathbb{E}\left[\sum_{t \in \mathcal{T}_{\Phi}} \hat{H}_{t}^{O P T}+\sum_{t \in \mathcal{T}_{\Psi}}\left(\tilde{H}_{t+1}^{O P T}+B_{t}^{O P T}\right)+\left(\sum_{t \in \mathcal{T}_{\Phi}} \hat{E}_{t}^{O P T}+\sum_{t \in \mathcal{T}_{\Psi}} \tilde{E}_{t+1}^{O P T}\right)\right] \\
\quad+\tilde{H}_{1}^{O P T}+\tilde{E}_{1}^{O P T} & 2 \mathbb{E}\left[\sum_{t=1}^{T}\left(\Phi_{t}^{O P T}+\Psi_{t}^{O P T}\right)+\tilde{H}_{1}^{O P T}+\tilde{E}_{1}^{O P T}\right] \\
= & 2 \mathbb{E}[\mathcal{C}(O P T)] .
\end{array}
$$

Hence, we have proved the following theorem, which provides a worst-case performance guarantee on the result of the MSMB policy.

THEOREM 2. The MSMB policy has a worst-case performance guarantee of two, i.e., for each instance of the backlogging model under service level constraints, the expected cost of the MSMB policy is at most twice the expected cost of an optimal solution, i.e., $\mathbb{E}[\mathcal{C}(M S M B)] \leq 2 \mathbb{E}[\mathcal{C}(O P T)]$. 


\section{Multi-Item Inventory Systems with Service Level Constraints}

\subsection{Model Setup and Bellman's Equations}

We extend our model in $\S 2$ by considering a multi-item inventory system where service level constraints are applied on the aggregate level. In our multi-item model, we consider a finite planning horizon of $T$ periods with $N$ items, indexed by $i=1, \ldots, N$. Similar as single-item model we described in $\S 2$, an information set $f_{t}$ is observed at the beginning of each period $t$ and contains all available information that can be used to predict future demands of all $N$ items, such as all past realized demand $d_{i, s}(1 \leq i \leq N, 1 \leq s<t)$ for item $i$ at period $s$. Let $x_{i, t}$ be the inventory position at the beginning of period $t$ for item $i, q_{i, t}$ be the production quantity for item $i$ in period $t$, and $y_{i, t}=x_{i, t}+q_{i, t}$ be the inventory position in period $t$ after production. Let $h_{i, t}$ and $b_{i, t}$ be the holding and backlogging cost for item $i$ at period $t$, then given lead time $L$, we can write the total holding and backlogging cost for item $i$ at period $t+L$ as

$$
G_{i, t}\left(y_{i, t}, f_{t}\right) \triangleq h_{i, t+L} \mathbb{E}\left[\left(y_{i, t}-D_{i,[t, t+L]}\right)^{+} \mid f_{t}\right]+b_{i, t+L} \mathbb{E}\left[\left(D_{i,[t, t+L]}-y_{i, t}\right)^{+} \mid f_{t}\right] .
$$

To define an appropriate service level constraint, we follow a similar modeling approach in Chen et al. (2017) and consider an aggregate service level constraint over all items.

For the $\alpha$ service level constraints, we have

$$
\mathbb{P}\left(\sum_{i=1}^{N} y_{i, t}-\sum_{i=1}^{N} D_{i,[t, t+L]} \geq 0 \mid f_{t}\right) \geq \theta_{t}, \quad \forall t=1, \ldots, T .
$$

For the $\beta$ service level constraints, we have

$$
\mathbb{E}\left[\frac{\left(\sum_{i=1}^{N}\left(y_{i, t}-D_{i,[t, t+L)}\right)^{+}\right.}{D_{t+L}} \mid f_{t}\right] \geq \tau_{t}, \quad \forall t=1, \ldots, T .
$$

Using the same argument as in $\S 2$, we can reduce either service level constraint (22) and (23) to a lower bound on the aggregate level of inventory position, i.e., $\sum_{i=1}^{N} y_{i, t} \geq r\left(f_{t}\right)$. Therefore, we can think the service level constraints as a lower bound on the aggregate total inventory position of $N$ items.

Let $v_{t}\left(\mathbf{x}_{t}, f_{t}\right)$ be the minimal expected cost from period $t+L$ to period $T+L$ given the inventory position $\mathbf{x}_{t}=\left\{x_{i, t}\right\}_{i=1}^{N}$ for each item $i=1,2, \ldots, N$ and the information set $f_{t} \in \mathcal{F}_{t}$ at the beginning of period $t$. We can use the following Bellman's equations to compute the optimal inventory policy:

$$
\begin{gathered}
v_{T+1}\left(\mathbf{x}_{T+1}, f_{T+1}\right)=0, \quad \forall x_{T+1} \in \mathbb{R}, f_{T+1} \in \mathcal{F}_{T+1}, \\
v_{t}\left(\mathbf{x}_{t}, f_{t}\right)=\min _{\mathbf{y}_{t} \geq \mathbf{x}_{t}, \sum_{i=1}^{N} y_{i, t} \geq r\left(f_{t}\right)}\left\{\sum_{i=1}^{N}\left(G_{i, t}\left(y_{i, t}, f_{t}\right)+c_{i, t} q_{i, t}\right)+\mathbb{E}\left[v_{t+1}\left(\mathbf{y}_{t}-\mathbf{D}_{t}, \mathcal{F}_{t+1}\right) \mid f_{t}\right]\right\}, \\
t=1, \ldots, T,
\end{gathered}
$$


where $c_{i, t}$ denotes the manufacturing cost for item $i$ in period $t$ and $\mathbf{D}_{t}=\left(D_{1, t}, \ldots, D_{N, t}\right)$ denotes the random demand for each item at period $t$ according to the information $f_{t}$ observed.

\subsection{A Heuristic Algorithm}

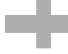

As already seen in $§ 3$, computing an exact optimal policy through a brute-force DP model (24) is generally intractable due to the curse of dimensionality. We describe a heuristic algorithm that can be used to solve (24) more efficiently. Without loss of generality, we assume that the unit production cost for each item in each period is zero following a standard cost transformation in the literature (see, e.g., Zipkin (2000)). This allows us to only consider holding costs and backlogging costs. Similar to the SMB algorithms presented in $\S 3$ and $\S 4$, we consider marginal costs of each item. The total marginal costs at period $t$ can be decomposed into the following parts:

- Marginal holding cost at period $t$ :

$$
M H_{t}\left(\mathbf{q}_{\mathbf{t}}\right)=\sum_{i=1}^{N} \sum_{j=t+L}^{T+L} h_{i, j}\left(\left(X_{i, t}+q_{i, t}-D_{i,[t, j]}\right)^{+}-\left(X_{i, t}-D_{i,[t, j]}\right)^{+}\right),
$$

where $X_{i, t}$ denotes a random inventory position which is realized at the beginning of period $t$.

- Marginal backlogging costs at period $t$ :

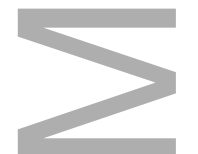

$$
M B_{t}\left(\mathbf{q}_{\mathbf{t}}\right)=\sum_{i=1}^{N} b_{i, t+L}\left(D_{i,[t, t+L]}-\left(q_{i, t}+X_{i, t}\right)\right)^{+} .
$$

The heuristic algorithm sequentially solves the following optimization problem for period $t=$ $1,2, \ldots, T$, given $\mathbf{x}_{\mathbf{t}}$ is the vector of initial inventory position at beginning of period $t$ for each item:

$$
\mathbf{q}_{\mathbf{t}}\left(\mathbf{x}_{\mathbf{t}}\right)=\underset{\mathbf{q}_{\mathbf{t}} \geq \mathbf{0}, \sum_{i=1}^{N}\left(x_{i, t}+q_{i, t}\right) \geq r\left(f_{t}\right)}{\arg \min } \mathbf{E}\left[M H_{t}\left(\mathbf{q}_{\mathbf{t}}\right)+M B_{t}\left(\mathbf{q}_{\mathbf{t}}\right) \mid f_{t}\right] .
$$

Since both marginal functions are convex in $\mathbf{q}_{\mathbf{t}},(27)$ is equivalent to minimizing an $N$-dimensional convex function, which is easy to implement using, e.g., the golden-section search method. We present our numerical experiments and results in $\S 6$.

\section{Numerical Experiments}

Since the remanufacturing model generalizes the classical backlogging model, we only focus on testing the MSMB policy and compare with the optimal policies derived through DP (for small problem sizes). Our numerical results show that the MSMB policy performs near-optimal for a set of instances with diverse demand and parameter settings. Moreover, the performance of the MSMB policy improves as we increase the levels of the QoS guarantee. 
Demand process. We consider the following three demand settings:

1. Independent and identically distributed (i.i.d.) demands. We test three specific demand distributions, namely, Exponential, Erlang-2, and Poisson all with mean equal to 10.

2. Markov-modulated demand process (MMDP). MMDP considers an underlying Markov Chain and assumes that the demand distribution depends on the state of the Markov Chain. The state at period $t$, denoted by $s_{t} \in\{1,2,3\}$ and is interpreted as the state of the economy (poor, fair or good). Given state $s_{t}$ at period $t$, the demand is a random variable with cumulative distribution function $F_{t}(\cdot)$ and mean value $\mu_{t}$. The better the state of economy, the larger the mean of the demand, i.e., $\mu_{1}<\mu_{2}<\mu_{3}$. The transition probability matrix is defined by $\mathbf{P}=\left(p_{i j}\right)_{3 \times 3}$, where $p_{i j}$ denotes the transition probability from state $s_{i}$ to state $s_{j}$. In our test data, the state of the economy follows a Markov chain with initial probabilities $p_{1}=p_{2}=p_{3}=1 / 3$ and transition probabilities

$$
\mathbf{P}=\left[\begin{array}{lll}
0.6 & 0.3 & 0.1 \\
0.2 & 0.6 & 0.2 \\
0.1 & 0.3 & 0.6
\end{array}\right]
$$

For each state $s_{t} \in\{1,2,3\}$, we also set the demand mean value as $5 s_{t}$ in period $t$.

We test three specific demand distributions, namely, Poisson, Uniform and Normal. The parameter of the Poisson distribution is solely governed by the mean value (set as 5,10, and 15). For the Uniformly distributed demand, we consider intervals $[0,10],[5,15]$ and $[10,20]$ for the three states, respectively. For the Normal distribution, we set the standard deviation $\sigma=2$ for all three states.

3. Autoregressive demands. For the autoregressive demand, we assume that there is a priori estimation $\mu_{t}$ of the demand at the period $t$. Besides, the realized demand also depends on the actual sales (or actual deviations from the priori) of previous seasons. Hence, the generic autoregressive demand model with parameter $\gamma$ has the following form:

$$
D_{t}-\mu_{t}=\sum_{s=t-\gamma}^{t-1} \psi_{t-s}\left(D_{s}-\mu_{s}\right)+\epsilon
$$

where $\psi_{t}$ stands for the extent of correlation for the demand deviations and $\epsilon$ is the noise term which is assumed to be a Gaussian white noise (i.e., standard normal distribution). The coefficients $\left\{\psi_{t}\right\}_{t=1}^{\gamma}$ are usually determined by the auto-covariances following the Yule-Walker Equations (cf. Hamilton 1994). Our numerical tests cover the following three cases:

- $\gamma=1$, with coefficients $\psi_{1}=1$;

- $\gamma=2$, with coefficients $\left(\psi_{1}, \psi_{2}\right)=(3 / 4,1 / 4)$;

- $\gamma=3$, with coefficients $\left(\psi_{1}, \psi_{2}, \psi_{3}\right)=(1 / 2,1 / 3,1 / 6)$; 
Return process. We consider two types of return process (a) independent product return with $U_{t}$ following a Normal distribution with mean $\mu=20$ and standard deviation $\sigma=5$; (b) dependent product return on the previous sales with $U_{t}=0.3 D_{t-1}+\nu_{t}$, where $\left\{\nu_{t}\right\}$ are i.i.d. Poisson random variables with rate 1 . We also cap the number of returned products by the number of items sold in the past in the second case (i.e., $\left.U_{t} \leq \sum_{i=1}^{t-1}\left(\min \left(d_{i}, q_{i}\right)-u_{i}\right), \forall t=1,2, \ldots, T\right)$.

Paramater settings. We consider a planning horizon $T=20$ periods and production lead time $L=2$. We assume that the cost parameters are stationary with a discounted factor $\alpha=0.99$ and a unit holding cost being normalized to 1 . We test different combinations of cost parameters under the three types of demand patterns. Specifically, we assume the unit remanufacturing cost $c_{1}=30$, the unit manufacturing cost $c_{2}=30,40,50$, and the unit backlogging cost $p=50,70,90$. For multi-item model, we assume the costs are the same among all three products.

Performance measure. To evaluate the performance of a policy $P$, we compare it with the results of the optimal policy. We use $\mathcal{C}(P)$ and $\mathcal{C}(O P T)$ to denote the costs given by the two policies, respectively. We define the suboptimality gap of the policy $P$ as the percentage of increase in the total cost of this policy compared to the optimal cost over the planning horizon, i.e.,

$$
\mathcal{E}=\frac{\mathcal{C}(P)-\mathcal{C}(O P T)}{\mathcal{C}(O P T)} \times 100 \%
$$

Clearly, the suboptimality gap $\mathcal{E}$ is always a positive number. Moreover, a smaller suboptimality gap means a better approximation algorithm. We report the values of $\mathcal{E}$ for every testing combination to empirically show that the proposed SMB policy provides close-to-optimal solutions in much more competitive CPU time. All of the numerical experiments are conducted on an $\operatorname{Intel}(\mathrm{R}) \mathrm{Xeon}(\mathrm{R})$ $2.93 \mathrm{GHz} \mathrm{PC}$ and we use Matlab R2013a as the solver.

\subsection{Numerical Results for the Single-Item Models}

Tables 1-6 present all the numerical results: Tables 1-3 cover the independent product return case and Tables 4-6 cover the dependent return case. For each instance, we test performance errors of the SMB policy for the i.i.d. demand, MMDP demand and autoregressive demand. Note that the average empirical suboptimality gap of the SMB policy is less than $2 \%$ in all instances, demonstrating the efficacy of the proposed approximation algorithm. Moreover, comparing the results of i.i.d., MMDP, and autoregressive demands, our algorithm performs consistently better in instances when demands are correlated. This indicates that the SMB policy takes advantage of given demand correlation information. On the other hand, the average CPU time of the SMB policy is around 1.16 seconds. In contrast, the DP algorithm for finding optimal solutions takes a much longer time (173.9 seconds on average) per test instance. 


\subsection{Numerical Results for the Multi-Item Model}

For the multi-item model, we tested the corresponding model with three items. The cost parameters are the same as in the single-item model and we also consider the aforementioned three demand processes (i.e., i.i.d., MMDP and Autoregressive). For each instance, we test performance errors of the heuristic policy against the optimal policy solved by dynamic program (24). From Tables 7-9, we observe that the average empirical suboptimality gap of the heuristic policy is less than $2 \%$ in all instances, demonstrating the efficacy of the proposed heuristic algorithm.

\begin{tabular}{|c|c|c|c|c|c|c|c|c|c|c|c|c|}
\hline \multirow{2}{*}{$\left(c_{1}, c_{2}, p\right)$} & \multicolumn{4}{|c|}{ Exponential } & \multicolumn{4}{|c|}{ Erlang-2 } & \multicolumn{4}{|c|}{ Poisson } \\
\hline & $\bar{\theta}=0.9$ & $=0.95$ & $\theta=0.98$ & $\theta=0.99$ & $\theta=0.9$ & $\theta=0.95$ & $\theta=0.98$ & $\theta=0.99$ & $\theta=0.9$ & $\theta=0.95$ & $\theta=0.98$ & $\theta=0.99$ \\
\hline$(30,30,50)$ & $1.17 \%$ & $0.95 \%$ & $1.40 \%$ & $1.07 \%$ & $0.52 \%$ & $0.24 \%$ & $0.49 \%$ & $0.81 \%$ & $0.08 \%$ & $0.21 \%$ & $0.36 \%$ & $0.57 \%$ \\
\hline$(30,30,70)$ & $0.97 \%$ & $0.81 \%$ & $1.11 \%$ & $1.09 \%$ & $1.13 \%$ & $0.48 \%$ & $0.42 \%$ & $0.94 \%$ & $0.04 \%$ & $0.09 \%$ & $0.24 \%$ & $0.49 \%$ \\
\hline$(30,30,90)$ & $1.39 \%$ & $0.65 \%$ & $0.77 \%$ & $1.43 \%$ & $1.44 \%$ & $0.69 \%$ & $0.21 \%$ & $0.56 \%$ & $0.11 \%$ & $0.07 \%$ & $0.25 \%$ & $0.43 \%$ \\
\hline$(30,40,50)$ & $0.64 \%$ & $1.11 \%$ & $0.75 \%$ & $0.65 \%$ & $0.80 \%$ & $0.46 \%$ & $0.57 \%$ & $0.82 \%$ & $0.06 \%$ & $0.15 \%$ & $0.40 \%$ & $0.58 \%$ \\
\hline$(30,40,70)$ & $0.91 \%$ & $0.70 \%$ & $1.24 \%$ & $1.61 \%$ & $0.97 \%$ & $0.56 \%$ & $0.31 \%$ & $0.53 \%$ & $0.08 \%$ & $0.11 \%$ & $0.33 \%$ & $0.51 \%$ \\
\hline$(30,40,90)$ & $0.76 \%$ & $0.84 \%$ & $1.35 \%$ & $1.40 \%$ & $1.41 \%$ & $0.72 \%$ & $0.38 \%$ & $0.41 \%$ & $0.10 \%$ & $0.07 \%$ & $0.24 \%$ & $0.41 \%$ \\
\hline$(30,50,50)$ & $1.28 \%$ & $0.99 \%$ & $0.94 \%$ & $1.01 \%$ & $0.73 \%$ & $0.19 \%$ & $0.46 \%$ & $0.95 \%$ & $0.04 \%$ & $0.11 \%$ & $0.37 \%$ & $0.55 \%$ \\
\hline$(30,5$ & $1.25 \%$ & $1.00 \%$ & $1.17 \%$ & $1.13 \%$ & $1.29 \%$ & $0.48 \%$ & $0.61 \%$ & $0.63 \%$ & $0.08 \%$ & $0.09 \%$ & $0.34 \%$ & $0.52 \%$ \\
\hline$(30,50,90)$ & $1.43 \%$ & $1.23 \%$ & $0.92 \%$ & $1.14 \%$ & $1.67 \%$ & $0.53 \%$ & $0.50 \%$ & $0.65 \%$ & $0.18 \%$ & $0.12 \%$ & $0.19 \%$ & $0.40 \%$ \\
\hline $\max$ & $1.43 \%$ & $1.23 \%$ & $1.4 \%$ & $1.61 \%$ & $1.67 \%$ & $0.72 \%$ & $0.61 \%$ & $0.95 \%$ & $0.18 \%$ & $0.21 \%$ & $0.40 \%$ & $0.58 \%$ \\
\hline mean & $1.09 \%$ & $0.92 \%$ & $1.07 \%$ & $1.17 \%$ & $1.11 \%$ & $0.48 \%$ & $0.44 \%$ & $0.70 \%$ & $0.09 \%$ & $0.11 \%$ & $0.30 \%$ & $0.50 \%$ \\
\hline
\end{tabular}

Table $1 \quad$ Suboptimality gap $\mathcal{E}$ for i.i.d. demands with different parameters (independent return)

\begin{tabular}{|c|c|c|c|c|c|c|c|c|c|c|c|c|}
\hline \multirow{2}{*}{$\left(c_{1}, c_{2}, p\right)$} & \multicolumn{4}{|c|}{ Poisson } & \multicolumn{4}{|c|}{ Uniform } & \multicolumn{4}{|c|}{ Normal } \\
\hline & $\theta=0$. & $=0.95$ & $\theta=0.98$ & $\theta=0.99$ & $\theta=0.9$ & $\theta=0.95$ & $\theta=0.98$ & $\theta=0.99$ & $\theta=0.9$ & $\theta=0.95$ & $\theta=0.98$ & $\theta=0.99$ \\
\hline$(30,30,50)$ & $0.42 \%$ & $0.16 \%$ & $0.66 \%$ & $0.85 \%$ & $0.39 \%$ & $0.35 \%$ & $0.71 \%$ & $0.90 \%$ & $0.20 \%$ & $0.37 \%$ & $0.65 \%$ & $0.74 \%$ \\
\hline$(30,30,70)$ & $0.77 \%$ & $0.29 \%$ & $0.37 \%$ & $0.74 \%$ & $0.55 \%$ & $0.34 \%$ & $0.61 \%$ & $0.83 \%$ & $0.27 \%$ & $0.25 \%$ & $0.55 \%$ & $0.76 \%$ \\
\hline$(30,30,90)$ & $0.95 \%$ & $0.39 \%$ & $0.49 \%$ & $0.55 \%$ & $0.72 \%$ & $0.37 \%$ & $0.54 \%$ & $0.82 \%$ & $0.40 \%$ & $0.28 \%$ & $0.49 \%$ & $0.76 \%$ \\
\hline$(30,40,50)$ & $0.39 \%$ & $0.30 \%$ & $0.64 \%$ & $0.79 \%$ & $0.40 \%$ & $0.42 \%$ & $0.72 \%$ & $0.88 \%$ & $0.21 \%$ & $0.29 \%$ & $0.61 \%$ & $0.79 \%$ \\
\hline$(30,40,70)$ & $0.65 \%$ & $0.31 \%$ & $0.51 \%$ & $0.61 \%$ & $0.56 \%$ & $0.41 \%$ & $0.67 \%$ & $0.87 \%$ & $0.28 \%$ & $0.26 \%$ & $0.56 \%$ & $0.73 \%$ \\
\hline$(30,40,90)$ & $0.79 \%$ & $0.30 \%$ & $0.40 \%$ & $0.58 \%$ & $0.65 \%$ & $0.38 \%$ & $0.54 \%$ & $0.84 \%$ & $0.41 \%$ & $0.24 \%$ & $0.43 \%$ & $0.73 \%$ \\
\hline$(30,50,50)$ & $0.46 \%$ & $0.25 \%$ & $0.50 \%$ & $0.95 \%$ & $0.40 \%$ & $0.42 \%$ & $0.75 \%$ & $0.87 \%$ & $0.21 \%$ & $0.30 \%$ & $0.62 \%$ & $0.82 \%$ \\
\hline$(30,50,70)$ & $0.63 \%$ & $0.27 \%$ & $0.43 \%$ & $0.65 \%$ & $0.56 \%$ & $0.35 \%$ & $0.62 \%$ & $0.84 \%$ & $0.28 \%$ & $0.27 \%$ & $0.61 \%$ & $0.75 \%$ \\
\hline$(30,50,90)$ & $0.98 \%$ & $0.39 \%$ & $0.43 \%$ & $0.59 \%$ & $0.64 \%$ & $0.34 \%$ & $0.55 \%$ & $0.77 \%$ & $0.36 \%$ & $0.27 \%$ & $0.49 \%$ & $0.68 \%$ \\
\hline $\max$ & $0.98 \%$ & $0.39 \%$ & $0.66 \%$ & $0.95 \%$ & $0.72 \%$ & $0.42 \%$ & $0.75 \%$ & $0.90 \%$ & $0.41 \%$ & $0.37 \%$ & $0.65 \%$ & $0.82 \%$ \\
\hline mean & $0.67 \%$ & $0.30 \%$ & $0.49 \%$ & $0.70 \%$ & $0.54 \%$ & $0.38 \%$ & $0.63 \%$ & $0.85 \%$ & $0.29 \%$ & $0.28 \%$ & $0.55 \%$ & $0.75 \%$ \\
\hline
\end{tabular}

Table $2 \quad$ Suboptimality gap $\mathcal{E}$ for MMDP demands with different parameters (independent return) 


\begin{tabular}{c|cccc|cccccccccc}
\hline \multirow{2}{*}{$\left(c_{1}, c_{2}, p\right)$} & \multicolumn{3}{|c|}{$\mathrm{AR}(1)$} & \multicolumn{4}{c|}{$\mathrm{AR}(2)$} & \multicolumn{4}{c}{ AR(3) } \\
\cline { 2 - 14 } & $\theta=0.9$ & $\theta=0.95$ & $\theta=0.98$ & $\theta=0.99$ & $\theta=0.9$ & $\theta=0.95$ & $\theta=0.98$ & $\theta=0.99$ & $\theta=0.9$ & $\theta=0.95$ & $\theta=0.98$ & $\theta=0.99$ \\
\hline$(30,30,50)$ & $0.26 \%$ & $0.30 \%$ & $0.52 \%$ & $0.71 \%$ & $0.22 \%$ & $0.35 \%$ & $0.54 \%$ & $0.71 \%$ & $0.20 \%$ & $0.36 \%$ & $0.58 \%$ & $0.67 \%$ \\
\hline$(30,30,70)$ & $0.39 \%$ & $0.33 \%$ & $0.44 \%$ & $0.62 \%$ & $0.33 \%$ & $0.31 \%$ & $0.49 \%$ & $0.64 \%$ & $0.24 \%$ & $0.31 \%$ & $0.50 \%$ & $0.64 \%$ \\
\hline$(30,30,90)$ & $0.59 \%$ & $0.38 \%$ & $0.46 \%$ & $0.59 \%$ & $0.51 \%$ & $0.33 \%$ & $0.54 \%$ & $0.62 \%$ & $0.31 \%$ & $0.34 \%$ & $0.43 \%$ & $0.63 \%$ \\
\hline$(30,40,50)$ & $0.27 \%$ & $0.34 \%$ & $0.55 \%$ & $0.73 \%$ & $0.24 \%$ & $0.31 \%$ & $0.57 \%$ & $0.68 \%$ & $0.18 \%$ & $0.30 \%$ & $0.57 \%$ & $0.68 \%$ \\
\hline$(30,40,70)$ & $0.46 \%$ & $0.30 \%$ & $0.42 \%$ & $0.63 \%$ & $0.30 \%$ & $0.33 \%$ & $0.52 \%$ & $0.66 \%$ & $0.26 \%$ & $0.32 \%$ & $0.46 \%$ & $0.66 \%$ \\
\hline$(30,40,90)$ & $0.64 \%$ & $0.38 \%$ & $0.43 \%$ & $0.67 \%$ & $0.41 \%$ & $0.33 \%$ & $0.47 \%$ & $0.58 \%$ & $0.34 \%$ & $0.31 \%$ & $0.44 \%$ & $0.66 \%$ \\
\hline$(30,50,50)$ & $0.29 \%$ & $0.30 \%$ & $0.58 \%$ & $0.73 \%$ & $0.24 \%$ & $0.32 \%$ & $0.49 \%$ & $0.68 \%$ & $0.20 \%$ & $0.32 \%$ & $0.60 \%$ & $0.70 \%$ \\
\hline$(30,50,70)$ & $0.45 \%$ & $0.32 \%$ & $0.49 \%$ & $0.71 \%$ & $0.31 \%$ & $0.33 \%$ & $0.50 \%$ & $0.68 \%$ & $0.24 \%$ & $0.32 \%$ & $0.50 \%$ & $0.63 \%$ \\
\hline$(30,50,90)$ & $0.63 \%$ & $0.35 \%$ & $0.43 \%$ & $0.60 \%$ & $0.42 \%$ & $0.33 \%$ & $0.46 \%$ & $0.63 \%$ & $0.30 \%$ & $0.33 \%$ & $0.46 \%$ & $0.63 \%$ \\
\hline max & $0.64 \%$ & $0.38 \%$ & $0.58 \%$ & $0.73 \%$ & $0.51 \%$ & $0.35 \%$ & $0.57 \%$ & $0.71 \%$ & $0.34 \%$ & $0.36 \%$ & $0.60 \%$ & $0.70 \%$ \\
\hline mean & $0.44 \%$ & $0.33 \%$ & $0.48 \%$ & $0.66 \%$ & $0.33 \%$ & $0.33 \%$ & $0.51 \%$ & $0.65 \%$ & $0.25 \%$ & $0.32 \%$ & $0.50 \%$ & $0.66 \%$ \\
\hline
\end{tabular}

Table 3 Suboptimality gap $\mathcal{E}$ for Autoregressive demands with different parameters (independent return)

\begin{tabular}{c|cccc|ccccc|cccc}
\hline \multirow{2}{*}{$\left(c_{1}, c_{2}, p\right)$} & \multicolumn{3}{|c|}{ Exponential } & \multicolumn{4}{|c|}{ Erlang-2 } & \multicolumn{4}{c}{ Poisson } \\
\cline { 2 - 14 } & $\theta=0.9$ & $\theta=0.95$ & $\theta=0.98$ & $\theta=0.99$ & $\theta=0.9$ & $\theta=0.95$ & $\theta=0.98$ & $\theta=0.99$ & $\theta=0.9$ & $\theta=0.95$ & $\theta=0.98$ & $\theta=0.99$ \\
\hline$(30,30,50)$ & $0.48 \%$ & $0.99 \%$ & $1.54 \%$ & $1.75 \%$ & $0.51 \%$ & $0.97 \%$ & $1.57 \%$ & $1.74 \%$ & $0.41 \%$ & $0.94 \%$ & $1.42 \%$ & $1.59 \%$ \\
\hline$(30,30,70)$ & $0.46 \%$ & $0.69 \%$ & $1.33 \%$ & $1.66 \%$ & $0.50 \%$ & $0.68 \%$ & $1.37 \%$ & $1.64 \%$ & $0.31 \%$ & $0.64 \%$ & $1.21 \%$ & $1.53 \%$ \\
\hline$(30,30,90)$ & $0.58 \%$ & $0.54 \%$ & $1.14 \%$ & $1.57 \%$ & $0.61 \%$ & $0.55 \%$ & $1.11 \%$ & $1.55 \%$ & $0.34 \%$ & $0.50 \%$ & $1.05 \%$ & $1.47 \%$ \\
\hline$(30,40,50)$ & $0.47 \%$ & $0.77 \%$ & $1.29 \%$ & $1.47 \%$ & $0.47 \%$ & $0.98 \%$ & $1.57 \%$ & $1.72 \%$ & $0.35 \%$ & $0.72 \%$ & $1.15 \%$ & $1.34 \%$ \\
\hline$(30,40,70)$ & $0.46 \%$ & $0.61 \%$ & $1.14 \%$ & $1.43 \%$ & $0.49 \%$ & $0.68 \%$ & $1.36 \%$ & $1.64 \%$ & $0.32 \%$ & $0.56 \%$ & $1.01 \%$ & $1.32 \%$ \\
\hline$(30,40,90)$ & $0.57 \%$ & $0.51 \%$ & $1.00 \%$ & $1.32 \%$ & $0.59 \%$ & $0.53 \%$ & $1.19 \%$ & $1.58 \%$ & $0.34 \%$ & $0.45 \%$ & $0.89 \%$ & $1.23 \%$ \\
\hline$(30,50,50)$ & $0.45 \%$ & $0.67 \%$ & $1.10 \%$ & $1.31 \%$ & $0.49 \%$ & $0.97 \%$ & $1.55 \%$ & $1.78 \%$ & $0.32 \%$ & $0.63 \%$ & $1.02 \%$ & $1.17 \%$ \\
\hline$(30,50,70)$ & $0.49 \%$ & $0.56 \%$ & $1.02 \%$ & $1.26 \%$ & $0.48 \%$ & $0.70 \%$ & $1.36 \%$ & $1.64 \%$ & $0.32 \%$ & $0.52 \%$ & $0.91 \%$ & $1.14 \%$ \\
\hline$(30,50,90)$ & $0.58 \%$ & $0.48 \%$ & $0.88 \%$ & $1.21 \%$ & $0.64 \%$ & $0.55 \%$ & $1.14 \%$ & $1.58 \%$ & $0.34 \%$ & $0.43 \%$ & $0.80 \%$ & $1.07 \%$ \\
\hline max & $0.58 \%$ & $0.99 \%$ & $1.54 \%$ & $1.75 \%$ & $0.64 \%$ & $0.98 \%$ & $1.57 \%$ & $1.78 \%$ & $0.41 \%$ & $0.94 \%$ & $1.42 \%$ & $1.59 \%$ \\
\hline mean & $0.50 \%$ & $0.65 \%$ & $1.16 \%$ & $1.44 \%$ & $0.53 \%$ & $0.73 \%$ & $1.36 \%$ & $1.65 \%$ & $0.34 \%$ & $0.60 \%$ & $1.05 \%$ & $1.32 \%$ \\
\hline
\end{tabular}

Table 4 Suboptimality gap $\mathcal{E}$ for i.i.d. demands with different parameters (dependent return)

\begin{tabular}{|c|c|c|c|c|c|c|c|c|c|c|c|c|}
\hline \multirow{2}{*}{$\left(c_{1}, c_{2}, p\right)$} & \multicolumn{4}{|c|}{ Poisson } & \multicolumn{4}{|c|}{ Uniform } & \multicolumn{4}{|c|}{ Normal } \\
\hline & $\theta=0$. & $\theta=0.95$ & $\theta=0.98$ & $\theta=0.99$ & $\theta=0.9$ & $\theta=0.95$ & $\theta=0.98$ & $\theta=0.99$ & $\theta=0.9$ & $\theta=0.95$ & $\theta=0.98$ & $\theta=0.99$ \\
\hline$(30,30,50)$ & $0.55 \%$ & $0.60 \%$ & $0.90 \%$ & $1.06 \%$ & $0.50 \%$ & $0.98 \%$ & $1.54 \%$ & $1.73 \%$ & $0.35 \%$ & $0.70 \%$ & $1.32 \%$ & $1.49 \%$ \\
\hline$(30,30,70)$ & $0.99 \%$ & $0.51 \%$ & $0.83 \%$ & $1.06 \%$ & $0.50 \%$ & $0.69 \%$ & $1.37 \%$ & $1.66 \%$ & $0.22 \%$ & $0.48 \%$ & $1.14 \%$ & $1.41 \%$ \\
\hline$(30,30,90)$ & $0.58 \%$ & $0.53 \%$ & $0.55 \%$ & $0.90 \%$ & $0.58 \%$ & $0.54 \%$ & $1.12 \%$ & $1.55 \%$ & $0.18 \%$ & $0.33 \%$ & $1.01 \%$ & $1.32 \%$ \\
\hline$(30,40,50)$ & $0.64 \%$ & $0.56 \%$ & $0.83 \%$ & $1.04 \%$ & $0.44 \%$ & $0.78 \%$ & $1.27 \%$ & $1.48 \%$ & $0.27 \%$ & $0.57 \%$ & $1.10 \%$ & $1.23 \%$ \\
\hline$(30,40,70)$ & $0.99 \%$ & $0.52 \%$ & $0.64 \%$ & $0.92 \%$ & $0.48 \%$ & $0.58 \%$ & $1.13 \%$ & $1.41 \%$ & $0.19 \%$ & $0.37 \%$ & $0.94 \%$ & $1.18 \%$ \\
\hline$(30,40,90)$ & $0.41 \%$ & $0.64 \%$ & $0.56 \%$ & $0.92 \%$ & $0.61 \%$ & $0.49 \%$ & $0.98 \%$ & $1.34 \%$ & $0.19 \%$ & $0.29 \%$ & $0.81 \%$ & $1.08 \%$ \\
\hline$(30,50,50)$ & $0.73 \%$ & $0.53 \%$ & $0.69 \%$ & $0.90 \%$ & $0.45 \%$ & $0.66 \%$ & $1.10 \%$ & $1.31 \%$ & $0.24 \%$ & $0.44 \%$ & $0.93 \%$ & $1.09 \%$ \\
\hline$(30,50,70)$ & $0.57 \%$ & $0.51 \%$ & $0.59 \%$ & $0.87 \%$ & $0.50 \%$ & $0.53 \%$ & $1.01 \%$ & $1.27 \%$ & $0.19 \%$ & $0.33 \%$ & $0.82 \%$ & $1.01 \%$ \\
\hline$(30,50,90)$ & $0.41 \%$ & $0.63 \%$ & $0.55 \%$ & $0.81 \%$ & $0.55 \%$ & $0.46 \%$ & $0.89 \%$ & $1.22 \%$ & $0.19 \%$ & $0.27 \%$ & $0.71 \%$ & $0.95 \%$ \\
\hline $\max$ & $0.99 \%$ & $0.64 \%$ & $0.90 \%$ & $1.06 \%$ & $0.61 \%$ & $0.98 \%$ & $1.54 \%$ & $1.73 \%$ & $0.35 \%$ & $0.70 \%$ & $1.32 \%$ & $1.49 \%$ \\
\hline mea & $0.65 \%$ & $0.56 \%$ & $0.68 \%$ & $0.94 \%$ & $0.51 \%$ & $0.64 \%$ & $1.16 \%$ & $1.44 \%$ & $0.23 \%$ & $0.42 \%$ & $0.97 \%$ & $1.20 \%$ \\
\hline
\end{tabular}

Table $5 \quad$ Suboptimality gap $\mathcal{E}$ for MMDP demands with different parameters (dependent return) 1

\section{Conclusion and Future Research Directions}

We have studied two stochastic inventory systems with probabilistic guarantees of service level (interpreted as stockout probabilities) in each period of a planning horizon. In particular, we have derived structural properties of optimal policies for both backlogging and remanufacturing models. We have also proposed several efficient and easily implementable approximation algorithms for computing near-optimal solutions, of which the efficacy is demonstrated through numerical experiments with diverse demand settings. 


\begin{tabular}{|c|c|c|c|c|c|c|c|c|c|c|c|}
\hline \multirow{2}{*}{$\left(c_{1}, c_{2}, p\right)$} & \multicolumn{3}{|c|}{$\operatorname{AR}(1)$} & \multicolumn{4}{|c|}{$\operatorname{AR}(2)$} & \multicolumn{4}{|c|}{$\overline{\mathrm{AR}}(3)$} \\
\hline & $\theta=0.9 \quad \theta=0.95$ & $\theta=0.98$ & $\theta=0.99$ & $=0.9$ & $\theta=0.95$ & $\theta=0.98$ & $\theta=0.99$ & $\theta=0.9$ & $\theta=0.95$ & $\theta=0.98$ & $\theta=0.99$ \\
\hline 50$)$ & $0.89 \%$ & $1.42 \%$ & $1.59 \%$ & $0.51 \%$ & $0.98 \%$ & $1.43 \%$ & $1.59 \%$ & $0.62 \%$ & $1.04 \%$ & $1.46 \%$ & $1.59 \%$ \\
\hline & $.69 \%$ & 1. & $\%$ & $48 \%$ & $5 \%$ & $3 \%$ & $3 \%$ & $0 \%$ & $5 \%$ & $\%$ & $1.52 \%$ \\
\hline 0$)$ & $69 \%$ & $\%$ & 0 & $49 \%$ & $0.66 \%$ & $1.12 \%$ & $1.42 \%$ & $.46 \%$ & $0.71 \%$ & $\%$ & $1.46 \%$ \\
\hline 50) & $0.70 \%$ & $1.14 \%$ & $1.32 \%$ & $0.42 \%$ & $0.74 \%$ & $1.16 \%$ & $1.32 \%$ & $0.46 \%$ & $0.79 \%$ & $1.19 \%$ & $1.31 \%$ \\
\hline$(30,4$ & $0.46 \%-0.56 \%$ & $1.02 \%$ & $1.25 \%$ & $0.39 \%$ & $0.61 \%$ & $1.05 \%$ & $1.23 \%$ & $0.42 \%$ & $0.67 \%$ & $1.10 \%$ & $1.26 \%$ \\
\hline$(30,40,90)$ & $0.55 \% \quad 0.51 \%$ & $0.86 \%$ & $1.18 \%$ & $0.44 \%$ & $0.55 \%$ & $0.93 \%$ & $1.19 \%$ & $0.41 \%$ & $0.59 \%$ & $0.96 \%$ & $1.22 \%$ \\
\hline & $0.39 \%$ & $0.99 \%$ & 1.1 & $0.38 \%$ & $0.62 \%$ & $1.00 \%$ & $1.14 \%$ & $0.42 \%$ & $0.65 \%$ & $1.01 \%$ & $1.13 \%$ \\
\hline & $0.43 \% \quad 0.50 \%$ & $0.88 \%$ & $1.12 \%$ & $0.37 \%$ & $0.52 \%$ & $0.91 \%$ & $1.10 \%$ & $0.38 \%$ & $0.59 \%$ & $0.94 \%$ & $1.10 \%$ \\
\hline$(30,50,90)$ & $0.51 \% \quad 0.46 \%$ & $0.78 \%$ & $1.02 \%$ & $0.43 \%$ & $0.51 \%$ & $0.81 \%$ & $1.06 \%$ & $0.39 \%$ & $0.53 \%$ & $0.85 \%$ & $1.04 \%$ \\
\hline & $0.69 \% \quad 0.89 \%$ & $1.42 \%$ & $1.59 \%$ & $0.51 \%$ & $0.98 \%$ & $1.43 \%$ & $1.59 \%$ & $0.62 \%$ & $1.04 \%$ & $1.46 \%$ & $1.59 \%$ \\
\hline mean & $0.50 \% \quad 0.61 \%$ & $1.05 \%$ & $1.29 \%$ & $0.44 \%$ & $0.66 \%$ & $1.08 \%$ & $1.28 \%$ & $0.45 \%$ & $0.71 \%$ & $1.11 \%$ & $1.29 \%$ \\
\hline
\end{tabular}

Table 6 Suboptimality gap $\mathcal{E}$ for Autoregressive demands with different parameters (dependent return)

\begin{tabular}{|c|c|c|c|c|c|c|c|c|c|c|c|c|}
\hline \multirow{2}{*}{$\left(c_{1}, c_{2}, p\right)$} & \multicolumn{4}{|c|}{ Exponential } & \multicolumn{4}{|c|}{ Erlang-2 } & \multicolumn{4}{|c|}{ Poisson } \\
\hline & $\theta=0.9$ & $=0.95$ & $\theta=0.98$ & $\theta=0.99$ & $\theta=0.9$ & $\theta=0.95$ & $\theta=0.98$ & $\theta=0.99$ & $\theta=0.9$ & $\theta=0.95$ & $\theta=0.98$ & $\theta=0.99$ \\
\hline$(30,30,50)$ & $1.14 \%$ & $0.98 \%$ & $1.42 \%$ & $1.17 \%$ & $0.57 \%$ & $0.34 \%$ & $0.44 \%$ & $0.71 \%$ & $0.1 \%$ & $0.26 \%$ & $0.46 \%$ & $0.47 \%$ \\
\hline$(30,30,70)$ & $0.88 \%$ & $0.89 \%$ & $1.04 \%$ & $1.05 \%$ & $1.12 \%$ & $0.52 \%$ & $0.46 \%$ & $0.97 \%$ & $0.08 \%$ & $0.19 \%$ & $0.32 \%$ & $0.38 \%$ \\
\hline$(30,30,90)$ & $1.29 \%$ & $0.68 \%$ & $0.79 \%$ & $1.35 \%$ & $1.41 \%$ & $0.65 \%$ & $0.23 \%$ & $0.55 \%$ & $0.13 \%$ & $0.06 \%$ & $0.21 \%$ & $0.41 \%$ \\
\hline$(30,40,50)$ & $0.56 \%$ & $1.04 \%$ & $0.79 \%$ & $0.69 \%$ & $0.76 \%$ & $0.43 \%$ & $0.48 \%$ & $0.79 \%$ & $0.09 \%$ & $0.12 \%$ & $0.38 \%$ & $0.49 \%$ \\
\hline$(30,40,70)$ & $0.95 \%$ & $0.68 \%$ & $1.19 \%$ & $1.57 \%$ & $0.99 \%$ & $0.59 \%$ & $0.33 \%$ & $0.49 \%$ & $0.09 \%$ & $0.11 \%$ & $0.32 \%$ & $0.56 \%$ \\
\hline$(30,40,90)$ & $0.76 \%$ & $0.81 \%$ & $1.32 \%$ & $1.51 \%$ & $1.51 \%$ & $0.79 \%$ & $0.36 \%$ & $0.44 \%$ & $0.14 \%$ & $0.09 \%$ & $0.27 \%$ & $0.42 \%$ \\
\hline$(30,50,50)$ & $1.25 \%$ & $0.95 \%$ & $0.94 \%$ & $1.04 \%$ & $0.78 \%$ & $0.22 \%$ & $0.43 \%$ & $0.98 \%$ & $0.08 \%$ & $0.17 \%$ & $0.38 \%$ & $0.45 \%$ \\
\hline$(30,50,70)$ & $1.18 \%$ & $0.98 \%$ & $1.13 \%$ & $1.11 \%$ & $1.28 \%$ & $0.56 \%$ & $0.64 \%$ & $0.67 \%$ & $0.12 \%$ & $0.16 \%$ & $0.33 \%$ & $0.46 \%$ \\
\hline$(30,50,90)$ & $1.40 \%$ & $1.27 \%$ & $0.96 \%$ & $1.18 \%$ & $1.70 \%$ & $0.73 \%$ & $0.49 \%$ & $0.62 \%$ & $0.13 \%$ & $0.07 \%$ & $0.23 \%$ & $0.36 \%$ \\
\hline $\max$ & $1.40 \%$ & $1.27 \%$ & $1.42 \%$ & $1.57 \%$ & $1.70 \%$ & $0.79 \%$ & $0.64 \%$ & $0.98 \%$ & $0.14 \%$ & $0.26 \%$ & $0.46 \%$ & $0.56 \%$ \\
\hline mean & $1.05 \%$ & $0.92 \%$ & $1.06 \%$ & $1.19 \%$ & $1.12 \%$ & $0.54 \%$ & $0.43 \%$ & $0.69 \%$ & $0.11 \%$ & $0.14 \%$ & $0.32 \%$ & $0.44 \%$ \\
\hline
\end{tabular}

Table $7 \quad$ Suboptimality gap $\mathcal{E}$ for i.i.d demands with different parameters (multi-item)

\begin{tabular}{|c|c|c|c|c|c|c|c|c|c|c|c|c|}
\hline \multirow{2}{*}{$\left(c_{1}, c_{2}, p\right)$} & \multicolumn{4}{|c|}{ Poisson } & \multicolumn{4}{|c|}{ Uniform } & \multicolumn{4}{|c|}{ Normal } \\
\hline & $\theta=0.9$ & $\theta=0.95$ & $\theta=0.98$ & $\theta=0.99$ & $\theta=0.9$ & $\theta=0.95$ & $\theta=0.98$ & $\theta=0.99$ & $\theta=0.9$ & $\theta=0.95$ & $\theta=0.98$ & $\theta=0.99$ \\
\hline$(30,30,50)$ & $0.47 \%$ & $0.13 \%$ & $0.67 \%$ & $0.89 \%$ & $0.49 \%$ & $0.38 \%$ & $0.77 \%$ & $0.98 \%$ & $0.29 \%$ & $0.34 \%$ & $0.59 \%$ & $0.71 \%$ \\
\hline$(30,30,70)$ & $0.77 \%$ & $0.38 \%$ & $0.39 \%$ & $0.77 \%$ & $0.59 \%$ & $0.32 \%$ & $0.54 \%$ & $0.81 \%$ & $0.31 \%$ & $0.29 \%$ & $0.57 \%$ & $0.69 \%$ \\
\hline$(30,30,90)$ & $0.91 \%$ & $0.46 \%$ & $0.49 \%$ & $0.58 \%$ & $0.77 \%$ & $0.45 \%$ & $0.54 \%$ & $0.81 \%$ & $0.46 \%$ & $0.32 \%$ & $0.53 \%$ & $0.72 \%$ \\
\hline$(30,40,50)$ & $0.39 \%$ & $0.30 \%$ & $0.63 \%$ & $0.77 \%$ & $0.44 \%$ & $0.38 \%$ & $0.69 \%$ & $0.85 \%$ & $0.25 \%$ & $0.24 \%$ & $0.56 \%$ & $0.73 \%$ \\
\hline$(30,40,70)$ & $0.78 \%$ & $0.36 \%$ & $0.55 \%$ & $0.64 \%$ & $0.46 \%$ & $0.48 \%$ & $0.78 \%$ & $0.84 \%$ & $0.21 \%$ & $0.25 \%$ & $0.57 \%$ & $0.67 \%$ \\
\hline$(30,40,90)$ & $0.78 \%$ & $0.24 \%$ & $0.48 \%$ & $0.55 \%$ & $0.60 \%$ & $0.37 \%$ & $0.52 \%$ & $0.84 \%$ & $0.43 \%$ & $0.29 \%$ & $0.36 \%$ & $0.70 \%$ \\
\hline$(30,50,50)$ & $0.47 \%$ & $0.22 \%$ & $0.52 \%$ & $0.99 \%$ & $0.42 \%$ & $0.46 \%$ & $0.74 \%$ & $0.83 \%$ & $0.28 \%$ & $0.42 \%$ & $0.56 \%$ & $0.77 \%$ \\
\hline$(30,50,70)$ & $0.65 \%$ & $0.29 \%$ & $0.38 \%$ & $0.56 \%$ & $0.65 \%$ & $0.42 \%$ & $0.55 \%$ & $0.75 \%$ & $0.34 \%$ & $0.41 \%$ & $0.56 \%$ & $0.73 \%$ \\
\hline$(30,50,90)$ & $0.91 \%$ & $0.42 \%$ & $0.48 \%$ & $0.72 \%$ & $0.66 \%$ & $0.31 \%$ & $0.58 \%$ & $0.73 \%$ & $0.39 \%$ & $0.25 \%$ & $0.46 \%$ & $0.74 \%$ \\
\hline $\max$ & $0.91 \%$ & $0.46 \%$ & $0.67 \%$ & $0.99 \%$ & $0.77 \%$ & $0.48 \%$ & $0.78 \%$ & $0.98 \%$ & $0.46 \%$ & $0.42 \%$ & $0.59 \%$ & $0.77 \%$ \\
\hline mean & $0.68 \%$ & $0.31 \%$ & $0.51 \%$ & $0.72 \%$ & $0.56 \%$ & $0.40 \%$ & $0.63 \%$ & $0.83 \%$ & $0.33 \%$ & $0.31 \%$ & $0.53 \%$ & $0.72 \%$ \\
\hline
\end{tabular}

Table $8 \quad$ Suboptimality gap $\mathcal{E}$ for MMDP demands with different parameters (multi-item)

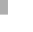

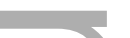

To close this paper, we point out two future research avenues. First, one may consider a joint service level constraint for restricting the stockout probability in any period over a finite time horizon. To the best of our knowledge, only Zhang et al. (2014) has considered a related dynamic lot-sizing problem with a joint chance constraint on stockout probability. The authors formulated as a multi-stage stochastic integer programming model solved by cutting-plane algorithms. There are no existing papers that characterized optimal or near-optimal policies for stochastic inventory models with joint service level constraints, which we believe is an important future research topic. 


\begin{tabular}{|c|c|c|c|c|c|c|c|c|c|c|c|c|}
\hline \multirow{2}{*}{$\left(c_{1}, c_{2}, p\right)$} & \multicolumn{4}{|c|}{$\mathrm{AR}(1)$} & \multicolumn{4}{|c|}{$\overline{\mathrm{AR}(2)}$} & \multicolumn{4}{|c|}{$\mathrm{AR}(3)$} \\
\hline & $=0.9$ & $\theta=0.95$ & $\theta=0.98$ & $\theta=0.99$ & $\theta=0.9$ & $\theta=0.95$ & $\theta=0.98$ & $\theta=0.99$ & $\theta=0.9$ & $\theta=0.95$ & $\theta=0.98$ & $\theta=0.99$ \\
\hline$(30,30,50)$ & $0.56 \%$ & $0.96 \%$ & $1.45 \%$ & $1.53 \%$ & $0.58 \%$ & $0.97 \%$ & $1.45 \%$ & $1.54 \%$ & $0.67 \%$ & $1.18 \%$ & $1.48 \%$ & $1.67 \%$ \\
\hline$(30,30,70)$ & $0.56 \%$ & $0.57 \%$ & $1.28 \%$ & $1.34 \%$ & $0.56 \%$ & $0.79 \%$ & $1.29 \%$ & $1.45 \%$ & $0.57 \%$ & $0.83 \%$ & $1.34 \%$ & $1.56 \%$ \\
\hline$(30,30,90)$ & $0.74 \%$ & $0.56 \%$ & $1.13 \%$ & $1.44 \%$ & $0.53 \%$ & $0.68 \%$ & $1.15 \%$ & $1.46 \%$ & $0.42 \%$ & $0.72 \%$ & $1.16 \%$ & $1.48 \%$ \\
\hline$(30,40,50)$ & $0.49 \%$ & $0.64 \%$ & $1.08 \%$ & $1.32 \%$ & $0.41 \%$ & $0.80 \%$ & $1.23 \%$ & $1.33 \%$ & $0.42 \%$ & $0.75 \%$ & $1.20 \%$ & $1.33 \%$ \\
\hline$(30,4$ & $0.45 \%$ & $0.52 \%$ & $1.13 \%$ & $1.35 \%$ & $0.42 \%$ & $0.61 \%$ & $1.07 \%$ & $1.14 \%$ & $0.47 \%$ & $0.68 \%$ & $1.13 \%$ & $1.27 \%$ \\
\hline$(30,40,90)$ & $0.51 \%$ & $0.50 \%$ & $0.89 \%$ & $1.17 \%$ & $0.48 \%$ & $0.53 \%$ & $0.97 \%$ & $1.24 \%$ & $0.45 \%$ & $0.66 \%$ & $0.90 \%$ & $1.24 \%$ \\
\hline$(30,50,50)$ & $0.38 \%$ & $0.59 \%$ & $1.21 \%$ & $1.19 \%$ & $0.39 \%$ & $0.65 \%$ & $1.03 \%$ & $1.11 \%$ & $0.45 \%$ & $0.63 \%$ & $1.08 \%$ & $1.14 \%$ \\
\hline$(30,50,70)$ & $0.42 \%$ & $0.56 \%$ & $0.83 \%$ & $1.16 \%$ & $0.39 \%$ & $0.59 \%$ & $0.96 \%$ & $1.10 \%$ & $0.37 \%$ & $0.64 \%$ & $0.93 \%$ & $1.18 \%$ \\
\hline$(30,50,90)$ & $0.53 \%$ & $0.47 \%$ & $0.78 \%$ & $1.10 \%$ & $0.45 \%$ & $0.57 \%$ & $0.79 \%$ & $1.03 \%$ & $0.38 \%$ & $0.52 \%$ & $0.88 \%$ & $1.06 \%$ \\
\hline & $0.74 \%$ & $0.96 \%$ & $1.45 \%$ & $1.53 \%$ & $0.58 \%$ & $0.97 \%$ & $1.45 \%$ & $1.54 \%$ & $0.67 \%$ & $1.18 \%$ & $1.48 \%$ & $1.67 \%$ \\
\hline mean & $0.52 \%$ & $0.60 \%$ & $1.09 \%$ & $1.29 \%$ & $0.47 \%$ & $0.69 \%$ & $1.10 \%$ & $1.27 \%$ & $0.47 \%$ & $0.73 \%$ & $1.12 \%$ & $1.33 \%$ \\
\hline
\end{tabular}

Table 9 Suboptimality gap $\mathcal{E}$ for Autoregressive demands with different parameters (multi-item)

Second, one may consider developing approximation algorithms for dual-sourcing problems (e.g., Xin and Goldberg (2017)), where the lead times of two sources are different.

\section{Acknowledgments.}

The authors thank the department editor Professor Qi Annabelle Feng, the anonymous associate editor, and the anonymous referees for their very constructive and detailed comments, which have helped significantly improve both the content and the exposition of this paper. The research of Yuchen Jiang and Cong Shi is partially supported by NSF grant CMMI-1634505. The research of Siqian Shen is partially supported by NSF grant CMMI-1433066.

\section{References}

Anderson ET, Fitzsimons GJ, Simester D (2006) Measuring and mitigating the costs of stockouts. Management Science 52(11):1751-1763.

Bertsimas D, Paschalidis IC (2001) Probabilistic service level guarantees in make-to-stock manufacturing systems. Operations Research 49(1):119-133.

Bookbinder JH, Tan JY (1988) Strategies for the probabilistic lot-sizing problem with service-level constraints. Management Science 34(9):1096-1108.

Boyaci T, Gallego G (2001) Serial production/distribution systems under service constraints. Manufacturing 8 Service Operations Management 3(1):43-50.

Chao X, Gong X, Shi C, Yang C, Zhang H, Zhou SX (2017) Approximation algorithms for capacitated perishable inventory systems with positive lead times. Management Science 64(11):5038-5061.

Chao X, Gong X, Shi C, Zhang H (2015) Approximation algorithms for perishable inventory systems. Operations Research 63(3):585-601.

Chen FY, Krass D (2001) Inventory models with minimal service level constraints. European Journal of Operational Research $134(1): 120-140$.

Chen L, Yücel Ş, Zhu K (2017) Inventory management in a closed-loop supply chain with advance demand information. Operations research letters 45(2):175-180.

Chen X, Hu P, He S (2013) Preservation of supermodularity in parametric optimization problems with nonlattice structures. Operations Research 61(5):1166-1173.

Cheung M, Elmachtoub A, Levi R, Shmoys DB (2016) The submodular joint replenishment problem. Mathematical Programming 158(1-2):207-233.

Cohen MA, Agrawal N, Agrawal V (2006) Winning in the aftermarket. Harvard business review 84(5):129.

Fitzsimons GJ (2000) Consumer response to stockouts. Journal of consumer research 27(2):249-266.

Gallego G, Özer Ö (2001) Integrating replenishment decisions with advance demand information. Management Science 47(10):1344-1360.

\section{This article is protected by copyright. All rights reserved}


Ghiani G, Laporte G, Musmanno R (2005) Introducing Logistics Systems (John Wiley \& Sons, Hoboken, NJ).

Glasserman P (2004) Monte Carlo Methods in Financial Engineering (Springer, New York, NY).

Goh J, Sim M (2011) Robust optimization made easy with rome. Operations Research 59(4):973-985.

Gong X, Chao X (2013) Optimal control policy for capacitated inventory systems with remanufacturing. Operations Research 61(3):603-611.

Graves S, Meal H, Dasu S, Qin Y (1986) Two-stage production planning in a dynamic environment. S. Axsater, C. Schneeweiss, E. Silver, eds. Multi-Stage Production Planning and Control. Lecture Notes in Economics and Mathematical Systems. Springer-Verlag, Berlin, Germany 9-43.

Halman N, Klabjan D, Mostagir M, Orlin J, Simichi-Levi D (2009) A fully polynomial time approximation scheme for single-item stochastic lot-sizing problems with discrete demand. Mathematics of Operations Research 34(3):674-685.

Hamilton JD (1994) Time series analysis (Princeton University Press, Princeton, NJ).

Heath DC, Jackson PL (1994) Modeling the evolution of demand forecasts with application to safety stock analysis in production/distribution system. IIE Transactions 26(3):17-30.

Jing X, Lewis M (2011) Stockouts in online retailing. Journal of Marketing Research 48(2):342-354.

Katok E, Thomas D, Davis A (2008) Inventory service-level agreements as coordination mechanisms: The effect of review periods. Manufacturing E Service Operations Management 10(4):609-624.

Levi R, Janakiraman G, Nagarajan M (2008a) A 2-approximation algorithm for stochastic inventory control models with lost sales. Mathematics of Operations Research 33(2):351-374.

Levi R, Pál M, Roundy RO, Shmoys DB (2007) Approximation algorithms for stochastic inventory control models. Mathematics of Operations Research 32(2):284-302.

Levi R, Roundy RO, Shmoys DB, Truong VA (2008b) Approximation algorithms for capacitated stochastic inventory control models. Operations Research 56(5):1184-1199.

Levi R, Shi C (2013) Approximation algorithms for the stochastic lot-sizing problem with order lead times. Operations Research 61(3):593-602.

Mills TC (1991) Time Series Techniques for Economists (Cambridge University Press, Cambridge, UK).

Nagarajan V, Shi C (2016) Approximation algorithms for inventory problems with submodular or routing costs. Mathematical Programming 160(1):225-244.

Nahmias S (1993) Production and operations analysis (Irwin, Homewood, IL).

Sethi SP, Cheng F (1997) Optimality of (s, S) policies in inventory models with markovian demand. Operations Research 45(6):931-939.

Shang KH, Song JS (2006) A closed-form approximation for serial inventory systems and its application to system design. Manufacturing \& Service Operations Management 8(4):394-406.

Simchi-Levi D, Chen X, Bramel J (2014) The Logic of Logistics: Theory, Algorithms, and Applications for Logistics and Supply Chain Management. Springer Series in Operations Research and Financial Engineering (Springer, New York, NY).

Simpson VP (1978) Optimum solution structure for a repairable inventory problem. Operations research 26(2):270-281.

Snyder LV, Shen ZJM (2011) Fundamentals of supply chain theory (John Wiley \& Sons, Hoboken, NJ).

Tao Z, Zhou SX (2014) Approximation balancing policies for inventory systems with remanufacturing. Mathematics of Operations Research 39(4):1179-1197.

Truong VA (2014) Approximation algorithm for the stochastic multiperiod inventory problem via a look-ahead optimization approach. Mathematics of Operations Research 39(4):1039-1056.

Wei L, Jasin S, Xin L (2018) Asymptotic optimality of order-up-to control for stochastic inventory systems with sequential probabilistic service level constraints, working Paper, University of Michigan, Ann Arbor, MI, USA.

Xin L, Goldberg DA (2017) Asymptotic optimality of tailored base-surge policies in dual-sourcing inventory systems. Management Science 64(1):437-452.

Zhang H, Shi C, Chao X (2016) Approximation algorithms for perishable inventory systems with setup costs. Operations Research 64(2):432-440.

Zhang M, Küçükyavuz S, Goel S (2014) A branch-and-cut method for dynamic decision making under joint chance constraints. Management Science 60(5):1317-1333.

Zhou SX, Tao Z, Chao X (2011) Optimal control of inventory systems with multiple types of remanufacturable products. Manufacturing \& Service Operations Management 13(1):20-34.

Zipkin P (2000) Foundations of Inventory Management (McGraw-Hill, New York, NY).

\section{This article is protected by copyright. All rights reserved}




\section{Appendix A: Technical Proofs for the Backlogging Model}

Proof of Proposition 1. This is a special case of Proposition 2.

Q.E.D.

Proof of Lemma 1. Let $\zeta_{t}$ be the random balanced cost by the SMB policy in period $t$, i.e., $\zeta_{t}=\mathbb{E}\left[\hat{H}_{t}^{S M B} \mid F_{t}\right]=\mathbb{E}\left[\tilde{H}_{t+1}^{S M B}+B_{t}^{S M B} \mid F_{t}\right]$. According to (13), we have

$$
\begin{aligned}
\mathbb{E}[\mathcal{C}(S M B)]= & \sum_{t=1}^{T} \mathbb{E}\left[\mathbb{E}\left[\hat{H}_{t}^{S M B}+\left(\tilde{H}_{t+1}^{S M B}+B_{t}^{S M B}\right) \mid F_{t}\right]\right]+\tilde{H}_{1}^{S M B} \\
= & 2 \sum_{t=1}^{T} \mathbb{E}\left[\zeta_{t}\right]+\tilde{H}_{1}^{S M B} \\
= & 2 \sum_{t} \mathbb{E}\left[\left(\mathbb{1}\left(t \in \mathcal{T}_{H}\right)+\mathbb{1}\left(t \in \mathcal{T}_{B}\right)\right) \cdot \zeta_{t}\right]+\tilde{H}_{1}^{S M B} \\
= & 2 \sum_{t} \mathbb{E}\left[E\left[\hat{H}_{t}^{S M B} \cdot \mathbb{1}\left(t \in \mathcal{T}_{H}\right) \mid F_{t}\right]\right] \\
& +2 \sum_{t} \mathbb{E}\left[\mathbb{E}\left[\left(\tilde{H}_{t+1}^{S M B}+B_{t}^{S M B}\right) \cdot \mathbb{1}\left(t \in \mathcal{T}_{B}\right) \mid F_{t}\right]\right]+\tilde{H}_{1}^{S M B} \\
= & 2 \mathbb{E}\left[\sum_{t \in \mathcal{T}_{H}} \hat{H}_{t}^{S M B}+\sum_{t \in \mathcal{T}_{B}}\left(\tilde{H}_{t+1}^{S M B}+B_{t}^{S M B}\right)\right]+\tilde{H}_{1}^{S M B} .
\end{aligned}
$$

Q.E.D.

Proof of Lemma 2. Suppose $t \in \mathcal{T}_{B}$, then $Y_{t}^{S M B} \geq Y_{t}^{O P T}$. Therefore,

$$
B_{t}^{S M B}=b_{t+L}\left(D_{[t, t+L]}-Y_{t}^{S M B}\right)^{+} \leq b_{t+L}\left(D_{[t, t+L]}-Y_{t}^{O P T}\right)^{+}=B_{t}^{O P T}
$$

Moreover, since $X_{t+1}^{S M B}=Y_{t}^{S M B}-D_{t} \geq Y_{t}^{O P T}-D_{t}=X_{t+1}^{O P T}$, we have

$$
\bar{X}_{t+1}^{S M B}-X_{t+1}^{S M B}=\left(r_{t+1}-X_{t+1}^{S M B}\right)^{+} \leq\left(r_{t+1}-X_{t+1}^{O P T}\right)^{+}=\bar{X}_{t+1}^{O P T}-X_{t+1}^{O P T} .
$$

Since Equation (8) is equivalent to

$$
\tilde{H}_{t}=\sum_{j=t+L}^{T+L} h_{j}\left(\bar{X}_{t}-X_{t}-\left(D_{[t, j]}-X_{t}\right)^{+}\right)^{+},
$$

we conclude that

$$
\begin{aligned}
\tilde{H}_{t+1}^{S M B} & =\sum_{j=t+1+L}^{T+L} h_{j}\left(\left(\bar{X}_{t+1}^{S M B}-X_{t+1}^{S M B}-\left(X_{t+1}^{S M B}-D_{[t+1, j]}\right)^{+}\right)\right)^{+} \\
& \leq \sum_{j=t+1+L}^{T+L} h_{j}\left(\left(\bar{X}_{t+1}^{O P T}-X_{t+1}^{O P T}-\left(X_{t+1}^{O P T}-D_{[t+1, j]}\right)^{+}\right)\right)^{+}=\tilde{H}_{t+1}^{O P T} .
\end{aligned}
$$

Q.E.D. 
Proof of Lemma 3. To show this, we prove the following inequality:

$$
\sum_{t \in \mathcal{T}_{H}}\left(\hat{H}_{t}^{S M B}+\tilde{H}_{t+1}^{S M B}\right) \leq \sum_{t \in \mathcal{T}_{H}}\left(\hat{H}_{t}^{O P T}+\tilde{H}_{t+1}^{O P T}\right)
$$

Since $\tilde{H}_{t+1}^{S M B} \geq \tilde{H}_{t+1}^{O P T}$ for any $t \in \mathcal{T}_{H}$ (following a similar argument in Lemma 2), we conclude that (28) implies our desired inequality.

Notice that for any policy $\mathrm{P}$, we have

$$
\begin{aligned}
\hat{H}_{t}^{P}+\tilde{H}_{t+1}^{P}= & \left.\sum_{j=t+L}^{T+L} h_{j}\left(\left(\bar{X}_{t}^{P}+\eta_{t}-D_{[t, j]}\right)^{+}-\left(\bar{X}_{t}^{P}-D_{[t, j]}\right)^{+}\right)\right\} \\
& +\sum_{j=t+L}^{T+L} h_{j}\left\{\left(\left(\bar{X}_{t+1}^{P}-D_{[t+1, j]}\right)^{+}-\left(X_{t+1}^{P}-D_{[t+1, j]}\right)^{+}\right)\right\} \\
= & \sum_{j=t+L}^{T+L} h_{j}\left\{\left(\bar{X}_{t+1}^{P}-D_{[t+1, j]}\right)^{+}-\left(\bar{X}_{t}^{P}-D_{[t, j]}\right)^{+}\right\}
\end{aligned}
$$

where the first equality applies the definition of $\hat{H}_{t}^{P}$ and $\tilde{H}_{t+1}^{P}$ (see (8) and (9)) and the second one cancels the first and the last terms using the system dynamic $X_{t+1}^{P}=\bar{X}_{t}^{P}+\eta_{t}^{P}-D_{t}$.

Suppose that $\mathcal{T}_{H}$ has $n$ intervals, i.e., $\mathcal{T}_{H}=I_{1} \cup I_{2} \cup \cdots \cup I_{n}$, where $I_{s}=\left[\xi_{s}^{1}, \xi_{s}^{2}\right]$. Then it suffices to show the desired inequality on each interval, i.e.,

$$
\sum_{t=\xi_{s}^{1}}^{\xi_{s}^{2}}\left(\hat{H}_{t}^{S M B}+\tilde{H}_{t+1}^{S M B}\right) \leq \sum_{t=\xi_{s}^{1}}^{\xi_{s}^{2}}\left(\hat{H}_{t}^{O P T}+\tilde{H}_{t+1}^{O P T}\right) .
$$

Let $\gamma_{s}(j)=\min \left\{j-L, \xi_{s}^{2}\right\}$. By interchanging the order of summation, we conclude that for each interval $I_{s}$ and any policy $\mathrm{P}$,

$$
\begin{aligned}
\sum_{t=\xi_{s}^{1}}^{\xi_{s}^{2}}\left(\hat{H}_{t}^{P}+\tilde{H}_{t+1}^{P}\right) & =\sum_{t=\xi_{s}^{1}}^{\xi_{s}^{2}} \sum_{j=t+L}^{T+L} h_{j}\left\{\left(\bar{X}_{t+1}^{P}-D_{[t+1, j]}\right)^{+}-\left(\bar{X}_{t}^{P}-D_{[t, j]}\right)^{+}\right\} \\
& =\sum_{j=\xi_{s}^{1}+L}^{T+L} h_{j} \sum_{t=\xi_{s}^{1}}^{\gamma_{s}(j)}\left\{\left(\bar{X}_{t+1}^{P}-D_{[t+1, j]}\right)^{+}-\left(\bar{X}_{t}^{P}-D_{[t, j]}\right)^{+}\right\} \\
& =\sum_{j=\xi_{s}^{1}+L}^{T+L} h_{j}\left\{\left(\bar{X}_{\gamma_{s}(j)+1}^{P}-D_{\left[\gamma_{s}(j)+1, j\right]}\right)^{+}-\left(\bar{X}_{\xi_{s}^{1}}^{P}-D_{\left[\xi_{s}^{1}, j\right]}\right)^{+}\right\} .
\end{aligned}
$$

For all $j=\xi_{s}^{1}+L, \ldots, T+L, \gamma_{s}(j) \in I_{s} \subseteq \mathcal{T}_{H}$, thus,

$$
X_{\gamma_{s}(j)+1}^{S M B}=Y_{\gamma_{s}(j)}^{S M B}-D_{\gamma_{s}(j)}<Y_{\gamma_{s}(j)}^{O P T}-D_{\gamma_{s}(j)}=X_{\gamma_{s}(j)+1}^{O P T}
$$

and consequently,

$$
\left(\bar{X}_{\gamma_{s}(j)+1}^{S M B}-D_{\left[\gamma_{s}(j)+1, j\right]}\right)^{+} \leq\left(\bar{X}_{\gamma_{s}(j)+1}^{O P T}-D_{\left[\gamma_{s}(j)+1, j\right]}\right)^{+}
$$


Also, since $\xi_{s}^{1}-1 \notin \mathcal{T}_{H}$, we obtain

$$
X_{\xi_{s}^{1}}^{S M B}=Y_{\xi_{s}^{1}-1}^{S M B}-D_{\xi_{s}^{1}-1} \geq Y_{\xi_{s}^{1}-1}^{O P T}-D_{\xi_{s}^{1}-1}=X_{\xi_{s}^{1}}^{O P T},
$$

(this inequality also holds when $\xi_{s}^{1}=1$ since $X_{1}^{S M B}=X_{1}^{O P T}$ ) and hence

$$
\left(\bar{X}_{\xi_{s}^{1}}^{S M B}-D_{\left[\xi_{s}^{1}, j\right]}\right)^{+} \geq\left(\bar{X}_{\xi_{s}^{1}}^{O P T}-D_{\left[\xi_{s}^{1}, j\right]}\right)^{+}
$$

Therefore,

$$
\begin{aligned}
\sum_{t=\xi_{s}^{1}}^{\xi_{s}^{2}}\left(\hat{H}_{t}^{S M B}+\tilde{H}_{t+1}^{S M B}\right) & =\sum_{j=\xi_{s}^{1}+L}^{T+L} h_{j}\left\{\left(\bar{X}_{\gamma_{s}(j)+1}^{S M B}-D_{\left[\gamma_{s}(j)+1, j\right]}\right)^{+}-\left(\bar{X}_{\xi_{s}^{1}}^{S M B}-D_{\left[\xi_{s}^{1}, j\right]}\right)^{+}\right\} \\
& \leq \sum_{j=\xi_{s}^{1}+L}^{T+L} h_{j}\left\{\left(\bar{X}_{\gamma_{s}(j)+1}^{O P T}-D_{\left[\gamma_{s}(j)+1, j\right]}\right)^{+}-\left(\bar{X}_{\xi_{s}^{1}}^{O P T}-D_{\left[\xi_{s}^{1}, j\right]}\right)^{+}\right\} \\
& =\sum_{t=\xi_{s}^{1}}^{\xi_{s}^{2}}\left(\hat{H}_{t}^{O P T}+\tilde{H}_{t+1}^{O P T}\right),
\end{aligned}
$$

where the first and the third equalities follow from (29), and the second inequality follows from (30) and (31).

Q.E.D.

\section{Appendix B: Technical Proofs for the Remanufacturing Model}

Proof of Proposition 2. For simplicity, we will omit the information $f_{t}$ in the proof. We change the decision variable from $\left(q_{t}^{1}, q_{t}^{2}\right)$ to $\left(q_{t}^{1}, y_{t}\right)$ where $y_{t}=x_{t}+q_{t}^{1}+q_{t}^{2}$ and define

$$
J_{t}\left(x_{t}, w_{t}, y_{t}, q_{t}^{1}\right)=\bar{G}_{t}\left(y_{t}\right)+\bar{c}_{t} q_{t}^{2}+\mathbb{E}\left[v_{t+1}\left(y_{t}-D_{t}, w_{t}-q_{t}^{1}+U_{t}\right)\right]
$$

for $t=1,2, \ldots, T$. Then the value function can be computed by

$$
v_{t}\left(x_{t}, w_{t}\right)=\min _{\left.q_{t}^{1}, y_{t} \in \mathcal{Y}\left(x_{t}, w_{t}, r_{t}\right)\right\}}\left\{J_{t}\left(x_{t}, w_{t}, y_{t}, q_{t}^{1}\right)\right\},
$$

where $\mathcal{Y}\left(x_{t}, w_{t}, r_{t}\right)=\left\{\left(q_{t}^{1}, y_{t}\right) \mid 0 \leq q_{t}^{1} \leq \min \left\{w_{t}, y_{t}-x_{t}\right\}, y_{t} \geq \max \left\{x_{t}, r_{t}\right\}\right\}$.

We first show that for all $f_{t} \in \mathcal{F}_{t}, v_{t}\left(x_{t}, w_{t}\right)$ is separable, convex in $\left(x_{t}, w_{t}\right)$ and linear and nonincreasing in $w_{t}$ with rate less than $\bar{c}_{t}$, i.e., there exists a convex function $z_{t}(\cdot)$ and a coefficient $\kappa_{t} \in\left[0, \bar{c}_{t}\right]$ such that $v_{t}\left(x_{t}, w_{t}\right)=z_{t}\left(x_{t}\right)-\kappa_{t} w_{t}$.

Clearly, when $t=T+1, v_{T+1}\left(x_{T+1}, w_{T+1}\right)=0$ satisfies these conditions.

Suppose the statement is true for $v_{t+1}\left(x_{t+1}, w_{t+1}\right)$, then we can write

$$
\begin{aligned}
\mathbb{E}\left[v_{t+1}\left(y_{t}-D_{t}, w_{t}-q_{t}^{1}+U_{t}\right)\right] & =\mathbb{E}\left[z_{t+1}\left(y_{t}-D_{t}\right)-\kappa_{t+1}\left(w_{t}-q_{t}^{1}+U_{t}\right)\right] \\
& =\tilde{z}_{t+1}\left(y_{t}\right)-\kappa_{t+1}\left(w_{t}-q_{t}^{1}\right)-\kappa_{t+1} \mathbb{E}\left[U_{t}\right]
\end{aligned}
$$


where $\tilde{z}_{t+1}(\cdot)$ is still a convex function. Hence, applying (32), we conclude that

$$
\begin{aligned}
J_{t}\left(x_{t}, w_{t}, y_{t}, q_{t}^{1}\right) & =\bar{G}_{t}\left(y_{t}\right)+\bar{c}_{t}\left(y_{t}-q_{t}^{1}-x_{t}\right)+\tilde{z}_{t+1}\left(y_{t}\right)-\kappa_{t+1}\left(w_{t}-q_{t}^{1}\right)-\kappa_{t+1} \mathbb{E}\left[U_{t}\right] \\
& =\left(\kappa_{t+1}-\bar{c}_{t}\right) q_{t}^{1}+\left(\bar{G}_{t}\left(y_{t}\right)+\tilde{z}_{t+1}\left(y_{t}\right)+\bar{c}_{t} y_{t}\right)-\bar{c}_{t} x_{t}-\kappa_{t+1}\left(w_{t}+\mathbb{E}\left[U_{t}\right]\right)
\end{aligned}
$$

Now we can compute $v_{t}\left(x_{t}, w_{t}\right)$ by first optimizing with respect to $q_{t}^{1}$. Since $\kappa_{t+1} \leq \bar{c}_{t+1} \leq \bar{c}_{t}$, the coefficient of $q_{t}^{1}$ is non-positive and hence $J_{t}\left(x_{t}, w_{t}, y_{t}, q_{t}^{1}\right)$ is non-increasing in $q_{t}^{1}$. Observing the feasible set $\mathcal{Y}\left(x_{t}, w_{t}, r_{t}\right)=\left\{\left(q_{t}^{1}, y_{t}\right) \mid 0 \leq q_{t}^{1} \leq w_{t}, y_{t} \geq x_{t}+q_{t}^{1}, y_{t} \geq r_{t}\right\}$, we conclude that $q_{t}^{1^{*}}=$ $\min \left\{w_{t}, y_{t}=x_{t}\right\}$.

- If $w_{t} \geq y_{t}-x_{t}, q_{t}^{1^{*}}=w_{t}$. Then

$$
J_{t}\left(x_{t}, w_{t}, y_{t}, q_{t}^{1^{*}}\right)=\left(\bar{G}_{t}\left(y_{t}\right)+\tilde{z}_{t+1}\left(y_{t}\right)+\bar{c}_{t} y_{t}\right)-\bar{c}_{t} w_{t}-\bar{c}_{t} x_{t}-\kappa_{t+1} \mathbb{E}\left[U_{t}\right]
$$

Since both $\bar{G}_{t}\left(y_{t}\right)$ and $\tilde{z}_{t+1}\left(y_{t}\right)$ are convex in $y_{t}$ we conclude that $J_{t}\left(x_{t}, w_{t}, y_{t}, q_{t}^{1^{*}}\right)$ is a convex function in $y_{t}$. Thus, if we define

$$
s_{t}=s\left(w_{t}, f_{t}\right)=\underset{y_{t}}{\arg \min }\left\{J_{t}\left(x_{t}, w_{t}, y_{t}, q_{t}^{1^{*}}\right)\right\}=\underset{y}{\arg \min }\left\{\bar{G}_{t}(y)+\tilde{z}_{t+1}(y)+\bar{c}_{t} y\right\}
$$

we conclude that $y_{t}^{*}=\max \left\{s_{t}, x_{t}, r_{t}\right\}$ minimizes $J_{t}\left(x_{t}, w_{t}, y_{t}, q_{t}^{1^{*}}\right)$ with respect to the feasible set $\mathcal{Y}\left(x_{t}, w_{t}, r_{t}\right)$. Moreover, $z_{t}\left(x_{t}\right)=\left(\bar{G}_{t}\left(y_{t}^{*}\right)+\tilde{z}_{t+1}\left(y_{t}^{*}\right)+\bar{c}_{t} y_{t}^{*}\right)-\bar{c}_{t} x_{t}-\kappa_{t+1} \mathbb{E}\left[U_{t}\right]$ must be a convex function in $x_{t}$. Hence, $v_{t}\left(x_{t}, w_{t}\right)=J_{t}\left(x_{t}, w_{t}, y_{t}^{*}, q_{t}^{1^{*}}\right)=z_{t}\left(x_{t}\right)-\bar{c}_{t} w_{t}$, where $z_{t}(\cdot)$ is a convex function.

- If $w_{t}<y_{t}-x_{t}, q_{t}^{1^{*}}=y_{t}-x_{t}$. In this case,

$$
J_{t}\left(x_{t}, w_{t}, y_{t}, q_{t}^{1^{*}}\right)=\left(\bar{G}_{t}\left(y_{t}\right)+\tilde{z}_{t+1}\left(y_{t}\right)+\kappa_{t+1} y_{t}\right)-\kappa_{t+1} w_{t}-\kappa_{t+1} x_{t}-\kappa_{t+1} \mathbb{E}\left[U_{t}\right]
$$

which is also convex in $y_{t}$. Hence, by defining

$$
s_{t}=s\left(w_{t}, f_{t}\right)=\underset{y_{t}}{\arg \min }\left\{J_{t}\left(x_{t}, w_{t}, y_{t}, q_{t}^{1^{*}}\right)\right\}=\underset{y}{\arg \min }\left\{\bar{G}_{t}(y)+\tilde{z}_{t+1}(y)+\kappa_{t+1} y\right\}
$$

we have $y_{t}^{*}=\max \left\{s_{t}, x_{t}, r_{t}\right\}$ minimizes $J_{t}\left(x_{t}, w_{t}, y_{t}, q_{t}^{1^{*}}\right)$. Moreover, $z_{t}\left(x_{t}\right)=\left(\bar{G}_{t}\left(y_{t}^{*}\right)+\tilde{z}_{t+1}\left(y_{t}^{*}\right)+\right.$ $\left.\kappa_{t+1} y_{t}^{*}\right)-\kappa_{t+1} x_{t}-\kappa_{t+1} \mathbb{E}\left[U_{t}\right]$ is convex in $x_{t}$ and $v_{t}\left(x_{t}, w_{t}\right)=J_{t}\left(x_{t}, w_{t}, y_{t}^{*}, q_{t}^{1^{*}}\right)=z_{t}\left(x_{t}\right)-\kappa_{t+1} w_{t}$, where $\kappa_{t+1} \leq \bar{c}_{t+1} \leq \bar{c}_{t}$.

Combining the two cases discussed above, we conclude that $v_{t}\left(x_{t}, w_{t}\right)=z_{t}\left(x_{t}\right)-\kappa_{t} w_{t}$ where $z_{t}(\cdot)$ is a convex function and $\kappa_{t} \in\left[0, c_{t}^{2}-c_{t}^{1}\right]$. Moreover, the minimizer $\left(y_{t}^{*}, q_{t}^{1^{*}}\right)$ must satisfy

$$
y_{t}^{*}=\max \left\{x_{t}, s_{t}, r_{t}\right\} ; \quad q_{t}^{1^{*}}=\min \left\{w_{t}, y_{t}^{*}-x_{t}\right\},
$$

where $s_{t}=s\left(w_{t}, f_{t}\right)=\arg \min _{y_{t}}\left\{J_{t}\left(x_{t}, w_{t}, y_{t}, q_{t}^{1^{*}}\right)\right\}$.

Q.E.D.

\section{This article is protected by copyright. All rights reserved}


Proof of Lemma 4. Let $\xi_{t}$ be the random balanced cost by the MSMB policy in period $t$, i.e., $\xi_{t}=\mathbb{E}\left[\Phi_{t}^{M S M B} \mid F_{t}\right]=\mathbb{E}\left[\Psi_{t}^{M S M B} \mid F_{t}\right]$. The total cost is computed by

$$
\begin{aligned}
\mathbb{E}[\mathcal{C}(M S M B)] & =\sum_{t=1}^{T} E\left[\Phi_{t}^{M S M B}+\Psi_{t}^{M S M B}\right]+\tilde{H}_{1}^{M S M B}+\tilde{E}_{1}^{M S M B} \\
& =\sum_{t=1}^{T} \mathbb{E}\left[\mathbb{E}\left[\Phi_{t}^{M S M B}+\Psi_{t}^{M S M B} \mid F_{t}\right]\right]+\tilde{H}_{1}^{M S M B}+\tilde{E}_{1}^{M S M B} \\
& =2 \sum_{t=1}^{T} \mathbb{E}\left[\xi_{t}\right]+\tilde{H}_{1}^{M S M B}+\tilde{E}_{1}^{M S M B} \\
& =2 \sum_{t} \mathbb{E}\left[\left(\mathbb{1}\left(t \in \mathcal{T}_{\Phi}\right)+\mathbb{1}\left(t \in \mathcal{T}_{\Psi}\right)\right) \cdot \xi_{t}\right]+\tilde{H}_{1}^{M S M B}+\tilde{E}_{1}^{M S M B} \\
& =2\left(\sum_{t} \mathbb{E}\left[E\left[\Phi_{t}^{M S M B} \cdot \mathbb{1}\left(t \in \mathcal{T}_{\Phi}\right) \mid F_{t}\right]\right]+\sum_{t} \mathbb{E}\left[\mathbb{E}\left[\Psi_{t}^{M S M B} \cdot \mathbb{1}\left(t \in \mathcal{T}_{\Psi}\right) \mid F_{t}\right]\right]\right) \\
& =2 \mathbb{E}\left[\sum_{t \in \mathcal{T}_{\Phi}} \Phi_{t}^{M S M B}+\sum_{t \in \mathcal{T}_{\Psi}} \Psi_{t}^{M S M B}\right]+\tilde{H}_{1}^{M S M B}+\tilde{E}_{1}^{M S M B} .
\end{aligned}
$$

Q.E.D.

Proof of Lemma 5. The proof is almost identical to that in Lemmas 2 and 3.

Q.E.D.

Proof of Lemma 6. For the first inequality, we have

$$
\begin{aligned}
X_{t+1}^{M S M B}+W_{t+1}^{M S M B} & =\bar{X}_{t}^{M S M B}+S_{t}^{M S M B}+\eta_{t}^{2, M S M B}-D_{t}+U_{t} \\
& \geq \bar{X}_{t}^{O P T}+S_{t}^{O P T}+\eta_{t}^{2, O P T}-D_{t}+U_{t} \\
& =X_{t+1}^{O P T}+W_{t+1}^{O P T}
\end{aligned}
$$

whenever $t \in \mathcal{T}_{c}$. Note that $(17)$ is equivalent to $\tilde{E}_{t}=\bar{c}_{t}\left(r_{t}-X_{t}-W_{t}\right)^{+}$. Therefore,

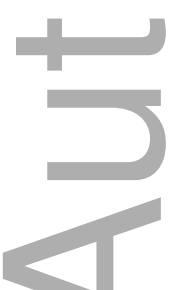

$$
\begin{aligned}
\tilde{E}_{t+1}^{M S M B} & =\bar{c}_{t+1}\left(r_{t+1}-X_{t+1}^{M S M B}-W_{t+1}^{M S M B}\right)^{+} \\
& \leq \bar{c}_{t+1}\left(r_{t+1}-X_{t+1}^{O P T}-W_{t+1}^{O P T}\right)^{+} \\
& =\tilde{E}_{t+1}^{O P T}
\end{aligned}
$$

for all $t \in \mathcal{T}_{c}$. Similarly, the inequality reverses when $t \in \mathcal{T}_{c}^{c}$.

Our second inequality is different from the one proved in Tao and Zhou (2014), in which they showed that the total production cost in periods $\mathcal{T}_{c}^{c}$ is less than that of the optimal policy.

For the second inequality, suppose that $\mathcal{T}_{c}^{c}$ has $n$ intervals, namely, $\mathcal{T}_{c}^{c}=I_{1} \cup I_{2} \cup \cdots \cup I_{n}$ where $I_{a}=\left[\xi_{a}^{1}, \xi_{a}^{2}\right]$. Then, we only need to show the inequality holds for each interval $I_{a}$. To see this, we 
compare the free-production level $\bar{X}_{t}+S_{t}$ between the MSMB and OPT policies. Given a policy $\mathrm{P}$, following the system dynamics in (21), we have

$$
\begin{aligned}
\bar{X}_{t}^{P}+S_{t}^{P} & =\bar{X}_{t}^{P}+\left(\left(S_{t-1}^{P}-\eta_{t-1}^{1, P}+U_{t-1}\right)-\left(\bar{X}_{t}^{P}-X_{t}^{P}\right)\right)^{+} \\
& =\max \left\{\bar{X}_{t}^{P}, S_{t-1}^{P}-\eta_{t-1}^{1, P}+X_{t}^{P}+U_{t-1}\right\} \\
& =\max \left\{\bar{X}_{t}^{P}, S_{t-1}^{P}+\bar{X}_{t-1}^{P}+\eta_{t-1}^{2, P}-D_{t-1}+U_{t-1}\right\} \\
& =\max \left\{r_{t}, S_{t-1}^{P}+\bar{X}_{t-1}^{P}+\eta_{t-1}^{2, P}-D_{t-1}+U_{t-1}\right\}
\end{aligned}
$$

where the last equality follows from $S_{t-1}+\bar{X}_{t-1}^{P}+\eta_{t-1}^{2, P}-D_{t-1} \geq X_{t}^{P}$ and $\bar{X}_{t}^{P}=\max \left\{X_{t}^{P}, r_{t}\right\}$. Therefore, for $t \in \mathcal{T}_{e}^{c}$,

$$
\begin{aligned}
\left(\bar{X}_{t+1}^{M S M B}+S_{t+1}^{M S M B}\right. & =\max \left\{r_{t+1}, S_{t}^{M S M B}+\bar{X}_{t}^{M S M B}+\eta_{t}^{2, M S M B}-D_{t}+U_{t}\right\} \\
& \leq \max \left\{r_{t+1}, S_{t}^{O P T}+\bar{X}_{t}^{O P T}+\eta_{t}^{2, O P T}-D_{t}+U_{t}\right\} \\
& =\bar{X}_{t+1}^{O P T}+S_{t+1}^{O P T}
\end{aligned}
$$

and similarly, $\bar{X}_{t+1}^{M S M B}+S_{t+1}^{M S M B} \geq \bar{X}_{t+1}^{O P T}+S_{t+1}^{O P T}$ for all $t \in \mathcal{T}_{e}$. Using the system dynamics, for any given policy $\mathrm{P}$, the additional manufacturing quantity is given by

$$
\eta_{t}^{2, P}=\left(X_{t+1}^{P}+W_{t+1}^{P}-\bar{X}_{t}^{P}-S_{t}^{P}\right)+\left(D_{t}-U_{t}\right)
$$

Thus for any $\beta \in I_{a}$,

$$
\begin{aligned}
\sum_{t=\xi_{a}^{1}}^{\beta} \eta_{t}^{2, P} & =\sum_{t=\xi_{a}^{1}}^{\beta}\left(X_{t+1}^{P}+W_{t+1}^{P}-\bar{X}_{t}^{P}-S_{t}^{P}\right)+\sum_{t=\xi_{a}^{1}}^{\beta}\left(D_{t}-U_{t}\right) \\
& =\sum_{t=\xi_{a}^{1}}^{\beta}\left(X_{t+1}^{P}+W_{t+1}^{P}-\bar{X}_{t+1}^{P}-S_{t+1}^{P}\right)+\left(\bar{X}_{\beta+1}^{P}+S_{\beta+1}^{P}\right)-\left(\bar{X}_{\xi_{a}^{1}}^{P}+S_{\xi_{a}^{1}}^{P}\right)+\sum_{t=\xi_{a}^{1}}^{\beta}\left(D_{t}-U_{t}\right) \\
& =-\sum_{t=\xi_{a}^{1}}^{\beta}\left(\bar{X}_{t+1}^{P}-X_{t+1}^{P}-W_{t+1}^{P}\right)^{+}+\left(\bar{X}_{\beta+1}^{P}+S_{\beta+1}^{P}\right)-\left(\bar{X}_{\xi_{a}^{1}}^{P}+S_{\xi_{a}^{1}}^{P}\right)+\sum_{t=\xi_{a}^{1}}^{\beta}\left(D_{t}-U_{t}\right) .
\end{aligned}
$$

Since $\beta \in \mathcal{T}_{c}^{c}$ and $\xi_{a}^{1}-1 \in \mathcal{T}_{c}$, we have $\bar{X}_{\beta+1}^{M S M B}+S_{\beta+1}^{M S M B} \leq \bar{X}_{\beta+1}^{O P T}+S_{\beta+1}^{O P T}$ and $\bar{X}_{\xi_{a}^{1}}^{M S M B}+S_{\xi_{a}^{1}}^{M S M B} \geq$ $\bar{X}_{\xi_{a}^{1}}^{O P T}+S_{\xi_{a}^{1}}^{O P T}$. Thus, from equality (33), we obtain

$$
\begin{aligned}
& \sum_{t=\xi_{a}^{1}}^{\beta}\left[\eta_{t}^{2, M S M B}+\left(\bar{X}_{t+1}^{M S M B}-X_{t+1}^{M S M B}-W_{t+1}^{M S M B}\right)^{+}\right] \\
= & \left(\bar{X}_{\beta+1}^{M S M B}+S_{\beta+1}^{M S M B}\right)-\left(\bar{X}_{\xi_{a}^{1}}^{M S M B}+S_{\xi_{a}^{1}}^{M S M B}\right)+\sum_{t=\xi_{a}^{1}}^{\beta}\left(D_{t}-U_{t}\right) \\
\leq & \left(\bar{X}_{\beta+1}^{O P T}+S_{\beta+1}^{O P T}\right)-\left(\bar{X}_{\xi_{a}^{1}}^{O P T}+S_{\xi_{a}^{1}}^{O P T}\right)+\sum_{t=\xi_{a}^{1}}^{\beta}\left(D_{t}-U_{t}\right)
\end{aligned}
$$




$$
=\sum_{t=\xi_{a}^{1}}^{\beta}\left[\eta_{t}^{2, O P T}+\left(\bar{X}_{t+1}^{O P T}-X_{t+1}^{O P T}-W_{t+1}^{O P T}\right)^{+}\right] .
$$

Note that $\eta_{t}^{2, M S M B}$ is the additional manufacturing quantity and $\left(\bar{X}_{t+1}^{M S M B}-X_{t+1}^{M S M B}-W_{t+1}^{M S M B}\right)^{+}$ is the forced manufacturing quantity; the above inequality allows us to compare the cumulative manufacturing quantity.

Following the assumption that the unit production cost $\bar{c}_{t}$ is non-increasing in $t$, we define $\Delta_{t}=\bar{c}_{t}-\bar{c}_{t+1}$ for all $t \in\left[\xi_{a}^{1}, \xi_{a}^{2}\right)$ and $\Delta_{\xi_{a}^{2}}=c_{\xi_{a}^{2}}$. Then since $\Delta_{t} \geq 0$ for all $t \in\left[\xi_{a}^{1}, \xi_{a}^{2}\right]$, by interchanging the order of summation, we conclude that

$$
\begin{aligned}
\sum_{t \in I_{a}}\left(\hat{E}_{t}^{M S M B}+\tilde{E}_{t+1}^{M S M B}\right) & =\sum_{t=\xi_{a}^{1}}^{\xi_{a}^{2}} \bar{c}_{t}\left[\eta_{t}^{2, M S M B}+\left(\bar{X}_{t+1}^{M S M B}-X_{t+1}^{M S M B}-W_{t+1}^{M S M B}\right)^{+}\right] \\
& =\sum_{t=\xi_{a}^{1}}^{\xi_{a}^{2}}\left(\sum_{\beta=t}^{\xi_{a}^{2}} \Delta_{\beta}\right)\left[\eta_{t}^{2, M S M B}+\left(\bar{X}_{t+1}^{M S M B}-X_{t+1}^{M S M B}-W_{t+1}^{M S M B}\right)^{+}\right] \\
& =\sum_{\beta=\xi_{a}^{1}}^{\xi_{a}^{2}} \Delta_{\beta} \sum_{t=\xi_{a}^{1}}^{\beta}\left[\eta_{t}^{2, M S M B}+\left(\bar{X}_{t+1}^{M S M B}-X_{t+1}^{M S M B}-W_{t+1}^{M S M B}\right)^{+}\right] \\
& \leq \sum_{\beta=\xi_{a}^{1}}^{\xi_{a}^{2}} \Delta_{\beta} \sum_{t=\xi_{a}^{1}}^{\beta}\left[\eta_{t}^{2, O P T}+\left(\bar{X}_{t+1}^{O P T}-X_{t+1}^{O P T}-W_{t+1}^{O P T}\right)^{+}\right] \\
& =\sum_{t \in I_{a}}\left(\hat{E}_{t}^{O P T}+\tilde{E}_{t+1}^{O P T}\right),
\end{aligned}
$$

which proves the second equality.

To show the last inequality, we first claim that for $\eta_{t}^{2, M S M B}=0$ for all $t \in \mathcal{T}_{\Phi} \cap \mathcal{T}_{c}$. Otherwise, suppose $\eta_{t}^{2, M S M B}>0$, then $\eta_{t}^{r, M S M B}=S_{t}^{M S M B}$. Thus,

$$
Y_{t}^{M S M B}=\bar{X}_{t}^{M S M B}+S_{t}^{M S M B}+\eta_{t}^{2, M S M B} \geq \bar{X}_{t}^{O P T}+S_{t}^{O P T}+\eta_{t}^{2, O P T} \geq Y_{t}^{O P T},
$$

which contradicts with $t \in \mathcal{T}_{\Phi}$. Similarly, we can show that $\eta_{t}^{2, O P T}=0$ holds for all $t \in \mathcal{T}_{\Psi} \cap \mathcal{T}_{c}^{c}$. Hence, using the second inequality we have proved above, we have

$$
\sum_{t \in \mathcal{T}_{\Phi}} \bar{c}_{t} \eta_{t}^{2, M S M B}=\sum_{t \in \mathcal{T}_{\Phi} \cap \mathcal{T}_{c}^{c}} \bar{c}_{t} \eta_{t}^{2, M S M B} \leq \sum_{t \in \mathcal{T}_{c}^{c}} \bar{c}_{t} \eta_{t}^{2, M S M B}
$$

and

$$
\sum_{t \in \mathcal{T}_{\Phi}} \bar{c}_{t} \eta_{t}^{2, O P T}=\sum_{t \in \mathcal{T}_{\Phi} \cup \mathcal{T}_{c}^{c}} \bar{c}_{t} \eta_{t}^{2, O P T} \geq \sum_{t \in \mathcal{T}_{c}^{c}} \bar{c}_{t} \eta_{t}^{2, O P T}
$$

Therefore, using the above inequalities together with the two inequalities we have already shown in the lemma, we conclude that

$$
\sum_{t \in \mathcal{T}_{\Phi}} \hat{E}_{t}^{M S M B}+\sum_{t \in \mathcal{T}_{\Psi}} \tilde{E}_{t+1}^{M S M B} \leq \sum_{t \in \mathcal{T}_{c}^{c}} \hat{E}_{t}^{M S M B}+\sum_{t \in \mathcal{T}_{\Psi} \cap \mathcal{T}_{c}^{c}} \tilde{E}_{t+1}^{M S M B}+\sum_{t \in \mathcal{T}_{\Psi} \cap \mathcal{T}_{c}} \tilde{E}_{t+1}^{M S M B}
$$




$$
\begin{aligned}
& =\sum_{t \in \mathcal{T}_{c}^{c}}\left(\hat{E}_{t}^{M S M B}+\tilde{E}_{t+1}^{M S M B}\right)-\sum_{t \in \mathcal{T}_{\Phi} \cap \mathcal{T}_{c}^{c}} \tilde{E}_{t+1}^{M S M B}+\sum_{t \in \mathcal{T}_{\Psi} \cap \mathcal{T}_{c}} \tilde{E}_{t+1}^{M S M B} \\
& \leq \sum_{t \in \mathcal{T}_{c}^{c}}\left(\hat{E}_{t}^{O P T}+\tilde{E}_{t+1}^{O P T}\right)-\sum_{t \in \mathcal{T}_{\Phi} \cap \mathcal{T}_{c}^{c}} \tilde{E}_{t+1}^{O P T}+\sum_{t \in \mathcal{T}_{\Psi} \cap \mathcal{T}_{c}} \tilde{E}_{t+1}^{O P T} \\
& =\sum_{t \in \mathcal{T}_{c}^{c}} \hat{E}_{t}^{O P T}+\sum_{t \in \mathcal{T}_{\Psi} \cap \mathcal{T}_{c}^{c}} \tilde{E}_{t+1}^{O P T}+\sum_{t \in \mathcal{T}_{\Psi} \cap \mathcal{T}_{c}} \tilde{E}_{t+1}^{O P T} \\
& \leq \sum_{t \in \mathcal{T}_{\Phi}} \hat{E}_{t}^{O P T}+\sum_{t \in \mathcal{T}_{\Psi} \cap \mathcal{T}_{c}^{c}} \tilde{E}_{t+1}^{O P T}+\sum_{t \in \mathcal{T}_{\Psi} \cap \mathcal{T}_{c}} \tilde{E}_{t+1}^{O P T} \\
& =\sum_{t \in \mathcal{T}_{\Phi}} \hat{E}_{t}^{O P T}+\sum_{t \in \mathcal{T}_{\Psi}} \tilde{E}_{t+1}^{O P T},
\end{aligned}
$$

where the first inequality follows from (34), the third inequality follows from the results proved in the first and second parts, and the fifth inequality follows from (35).

Q.E.D.

\section{Appendix C: An Equivalent Random Capacity Model with Two Suppliers}

Consider a single-product inventory system that orders from two suppliers, namely, a regular supplier and a discounted (but random) supplier, over a planning horizon of $T$ periods, indexed by $t=1, \ldots, T$. Both suppliers have the same lead times $L$.

The sequence of events is as follows. In each period $t$, the decision maker reviews the starting inventory level of the inventory system, and also queries the available inventory from the discounted supplier. The regular supplier, on the other hand, has unlimited capacity. However, the unit cost from regular supplier $c_{t}^{2}$ is higher than the unit cost from discounted supplier $c_{t}^{1}$. Then the decision maker decides how many units to order from the regular supplier, denoted by $q_{t}^{2}$, and how many units to order from the discounted supplier, denoted by $q_{t}^{1}$. Then the random customer demand $D_{t}=d_{t}$ is realized. Also, the discounted supplier obtains a random amount of inventory $U_{t}=u_{t}$ and then adds to her own available inventory. Finally, all costs (including unit costs from both suppliers, holding and backlogging costs) for this period are assessed. Note that we use $h_{t}^{2}$ to denote the unit holding cost for each on-hand inventory and $b_{t}$ to denote the unit backlogging cost. Moreover, to incentivize the discounted supplier to reserve her inventory for the decision maker (at a lower unit price), the decision maker needs to compensate $h_{t}^{1}$ per unit of the inventory that the discounted supplier is holding. On the other hand, the regular supplier needs no compensation.

From the model description, it is evident that this random capacity model with two suppliers is equivalent to the original remanufacturing model. 
This article is protected by copyright. All rights reseryed

$q_{t}^{D B}\left(r_{t}-x_{t}\right)^{+} \quad$ Ordering quantity $\left(q_{t}\right)$

Additional ordering quantity $\left(\eta_{t}\right)$ 


$$
H_{t}\left(\eta_{t}\right) \stackrel{\prime}{=} \hat{H}_{t}\left(\eta_{t}\right)+\tilde{H}_{t}\left(\eta_{t}\right)
$$

$\tilde{H}_{t}\left(\eta_{t}\right)$

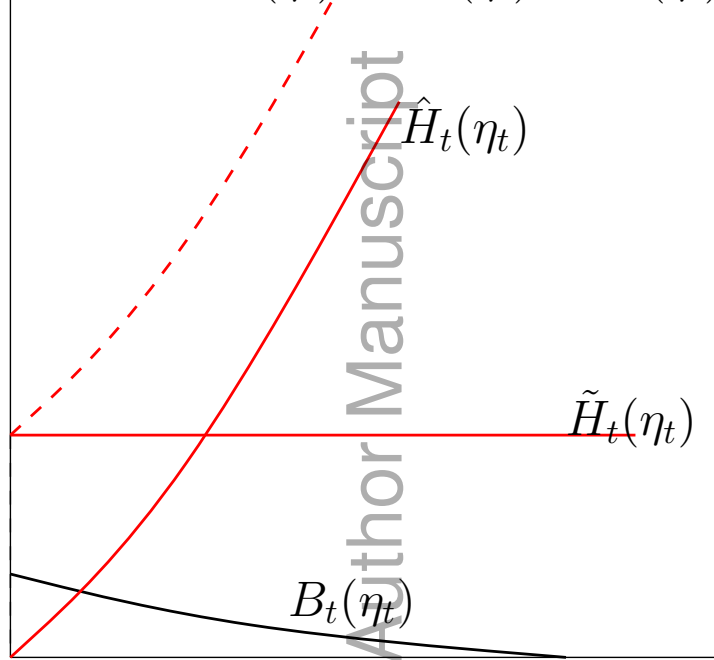


